\title{
COMPUTABLE ERROR ESTIMATES FOR FINITE ELEMENT APPROXIMATIONS OF ELLIPTIC PARTIAL DIFFERENTIAL EQUATIONS WITH ROUGH STOCHASTIC DATA*
}

\author{
ERIC JOSEPH HALL ${ }^{\dagger}$, HÅKON HOEL ${ }^{\ddagger}$, MATTIAS SANDBERG§ ${ }^{\S}$ ANDERS SZEPESSY ${ }^{\S}$, \\ AND RAÚL TEMPONE ${ }^{\uparrow}$
}

\begin{abstract}
We derive computable error estimates for finite element approximations of linear elliptic partial differential equations (PDE) with rough stochastic coefficients. In this setting, the exact solutions contain high frequency content that standard a posteriori error estimates fail to capture. We propose goal-oriented estimates, based on local error indicators, for the pathwise Galerkin and expected quadrature errors committed in standard, continuous, piecewise linear finite element approximations. Derived using easily validated assumptions, these novel estimates can be computed at a relatively low cost and have applications to subsurface flow problems in geophysics where the conductivities are assumed to have lognormal distributions with low regularity. Our theory is supported by numerical experiments on test problems in one and two dimensions.
\end{abstract}

Key words. a posteriori error, Galerkin error, quadrature error, elliptic PDE, random PDE, Monte Carlo methods, lognormal

AMS subject classifications. 60H35 (Primary), 65N15, 35R60 (Secondary)

1. Introduction. There is a vast body of literature concerning a posteriori error estimation of finite element approximations of elliptic PDE. All of these works, to some extent, examine models in which the conductivities enjoy a certain degree of smoothness. Motivated by problems arising in geophysics, we present computable error estimates for piecewise linear finite element approximations of a class of elliptic problems where the conductivities are modeled by rough stochastic processes. In this setting, the typical a posteriori error analysis does not yield computable estimates.

To understand where the standard analysis breaks down, it is instructive to consider the following homogeneous two-point boundary value problem:

$$
-\left(a(\omega, x) u^{\prime}(\omega, x)\right)^{\prime}=0
$$

$P$-a.s. for $x \in[0,1]$ subject to the boundary conditions $u(0)=u(\omega, 0)=0$ and $a(1) u^{\prime}(1)=a(\omega, 1) u^{\prime}(\omega, 1)=1, P$-a.s., where $\omega \in \Omega$ for a given probability space $(\Omega, \mathbb{F}, P)$ and where $u^{\prime}$ denotes the derivative of $u$ with respect to $x$. We seek a solution, $u=u(\omega, \cdot)$, to this equation, parameterized by $\omega$, in the Sobolev space $\mathcal{H}^{1}(0,1)$ for a given process $a(x)=a(\omega, x)=e^{B(\omega, x)}$ where $(B(\omega, x))_{x \in[0,1]}$ is a Brownian bridge pegged to zero at the end points. By Kolmogorov's continuity theorem [35], $B(x)$ admits a version that is Hölder continuous with exponent $1 / 2-\varepsilon$ for $\varepsilon \in(0,1 / 2)$

* The research was supported by the Swedish Research Council grant VR-621-2014-4776, and the Swedish e-Science Research Center. It was carried out while E.J. Hall was a Göran Gustafsson postdoctoral fellow at KTH Royal Institute of Technology. R. Tempone is a member of the KAUST Strategic Research Initiative, Center for Uncertainty Quantification in Computational Sciences and Engineering. R. Tempone received support from the KAUST CRG3 Award Ref: 2281. H. Hoel received support by Norges Forskningsråd, research project 214495 LIQCRY.

${ }^{\dagger}$ Department of Mathematics and Statistics, University of Massachusetts Amherst, Amherst, MA 10030, United States (hall@math.umass.edu).

$\ddagger$ Department of Mathematics, University of Oslo, 0316 Oslo, Norway (haakonah@math.uio.no).

$\S$ Department of Mathematics, KTH Royal Institute of Technology, 10044 Stockholm, Sweden (msandb@kth.se, szepessy@kth.se).

$\S$ Applied Mathematics and Computational Science, King Abdullah University of Science and Technology, Thuwal, 23955-6900, Kingdom of Saudi Arabia. (raul.tempone@kaust.edu.sa). 
and thus $a$ admits a version $a \in C^{\frac{1}{2}-\varepsilon}([0,1])$. In what follows we take $a$ to be this almost $1 / 2$-Hölder continuous version and we note that $a(0)=a(1)=1$ for almost all $\omega \in \Omega$. As a matter of course, one cannot expect much smoothness from the solution: we have $u \in C^{\frac{3}{2}-\varepsilon}([0,1])$, for $\varepsilon \in(0,1 / 2)$, since $u^{\prime}(x)=1 / a(x)$.

Our goal is to determine moments of an observable $(u, g)$, a linear functional of the solution to (1.1), for a given $g$. The problem is to estimate the expected error in the observable,

$$
\mathbf{E}\left[\left(u-\bar{u}_{h}, g\right)\right]=\mathbf{E}\left[\int_{0}^{1}\left(u-\bar{u}_{h}\right)(x) g(x) \mathrm{d} x\right],
$$

where $\bar{u}_{h}$ is the finite element approximation with a given quadrature of the bilinear form, for a given $\omega$, taking values in $V_{h}$, the space of piecewise linear elements. The error can be split into a part corresponding to the Galerkin error

$$
\mathbf{E}\left[\left(u-u_{h}, g\right)\right]=\mathbf{E}\left[\int_{0}^{1}\left(u-u_{h}\right)(x) g(x) \mathrm{d} x\right],
$$

and another part corresponding to the quadrature error

$$
\mathbf{E}\left[\left(u_{h}-\bar{u}_{h}, g\right)\right]=\mathbf{E}\left[\int_{0}^{1}\left(u_{h}-\bar{u}_{h}\right)(x) g(x) \mathrm{d} x\right],
$$

where $u_{h}$ solves the finite element problem with exact quadrature. In the case of finite element approximations of problems with smooth conductivity functions $a$, the quadrature error can be made small compared to the Galerkin error with asymptotically negligible additional work. In the case of conductivities with low regularity, the situation is different: we observe that, under mild assumptions, the pathwise quadrature error is typically much larger than the Galerkin error and the expected value of the quadrature error, proved in Theorem 6.1, is of the same order as the Galerkin error, in the sense that doubling the number of quadrature points reduces the expected quadrature error with the same factor as the Galerkin error when doubling the number of nodal points. Therefore the quadrature error is at least as important as the Galerkin error when analyzing the discretization error for problems where the conductivity has low regularity. Indeed, if the regularity is lower than the rough lognormal conductivity considered in (1.1), then the quadrature error may dominate. The pathwise Galerkin error, derived in Theorem 4.1, is in some sense simpler to analyze. We observe that the pathwise Galerkin error is of the same order as the expected value of the Galerkin error, suggesting that there are no large stochastic cancellations present, in contrast to the situation for the quadrature error. Nevertheless, the low regularity of the conductivity makes our analysis of the Galerkin error different from the typical a posteriori analysis.

To motivate why the standard a posteriori analysis of Galerkin errors is not directly applicable in the case of conductivities with low regularity, we sketch the classical a posteriori error analysis in the energy norm for the model problem (1.1). We begin by recalling a classical result in [3] that gives an a priori bound for the finite element approximation in the energy norm,

$$
\|w\|_{E}:=\left(\int_{0}^{1} a(x)\left(w^{\prime}(x)\right)^{2} \mathrm{~d} x\right)^{\frac{1}{2}},
$$


for elliptic PDE (analogous estimates for elliptic PDE with lognormal coefficients, such as those given in $[12,13]$, are discussed further in $\S 2$ ). Namely, for $s<1 / 2$,

$$
\left\|u-u_{h}\right\|_{E} \leq \inf _{v \in V_{h}}\|u-v\|_{E} \lesssim h^{s}\|u\|_{1+s}
$$

estimates the Galerkin error committed pathwise assuming that the quadrature used in computing $u_{h}$ is exact. Here $\|\cdot\|_{1+s}$ denotes the norm in the Sobolev space $\mathcal{H}^{1+s}(0,1)$ of functions with up to $1+s$ derivatives in $L^{2}(0,1)$ (for a definition of such spaces see [11]) and we observe that $u \in C^{\frac{3}{2}-\varepsilon}([0,1])$ so that also $u \in \mathcal{H}^{\frac{3}{2}-\varepsilon}(0,1)$. Next, we rewrite the pathwise error functional for (1.2) as

$$
\int_{0}^{1} a(x)\left(u^{\prime}-u_{h}^{\prime}\right)(x)\left(\lambda^{\prime}-\lambda_{h}^{\prime}\right)(x) \mathrm{d} x
$$

by introducing the dual problem in the variable $\lambda$ :

$$
-\left(a(\omega, x) \lambda^{\prime}(\omega, x)\right)^{\prime}=g(x),
$$

$P$-a.s. for $x \in[0,1]$, subject to the boundary conditions $\lambda(\omega, 0)=\lambda^{\prime}(\omega, 1)=0, P$-a.s. The convergence of the pathwise Galerkin error is on the order

$$
\left(u-u_{h}, g\right) \leq\left\|u-u_{h}\right\|_{E}\left\|\lambda-\lambda_{h}\right\|_{E}=O\left(h^{2 s}\right),
$$

using (1.3) for sufficiently regular $g$. The error in the energy norm is

$$
\begin{aligned}
\left\|u-u_{h}\right\|_{E}^{2}= & \int_{0}^{1} a\left(u^{\prime}-u_{h}^{\prime}\right)\left(u-u_{h}\right)^{\prime} \mathrm{d} x=\int_{0}^{1} a\left(u^{\prime}-u_{h}^{\prime}\right)\left(u-u_{h}-v\right)^{\prime} \mathrm{d} x \\
= & \sum_{k=0}^{N-1} \int_{x_{k}}^{x_{k+1}} a\left(u^{\prime}-u_{h}^{\prime}\right)\left(u-u_{h}-v\right)^{\prime} \mathrm{d} x \\
= & \sum_{k=0}^{N-1}\left(\int_{x_{k}}^{x_{k+1}}-\left(a\left(u^{\prime}-u_{h}^{\prime}\right)\right)^{\prime}\left(u-u_{h}-v\right) \mathrm{d} x\right. \\
& \left.+\left[a\left(u^{\prime}-u_{h}^{\prime}\right)\left(u-u_{h}-v\right)\right]_{x_{k}}^{x_{k+1}}\right) \\
= & \sum_{k=0}^{N-1}\left(\int_{x_{k}}^{x_{k+1}}(\underbrace{\left(-a u^{\prime}\right)^{\prime}}_{=0}+\left(a u_{h}^{\prime}\right)^{\prime})\left(u-u_{h}-v\right) \mathrm{d} x\right. \\
& \left.+\left[a\left(u^{\prime}-u_{h}^{\prime}\right)\left(u-u_{h}-v\right)\right]_{x_{k}}^{x_{k+1}}\right) \\
= & \sum_{k=0}^{N-1} \int_{x_{k}}^{x_{k+1}}\left(a u_{h}^{\prime}\right)^{\prime}\left(u-u_{h}-v\right) \mathrm{d} x \\
& +\sum_{k=1}^{N-1} a\left(x_{k}\right) \underbrace{\left(u_{h}^{\prime}\left(x_{k}^{+}\right)-u_{h}^{\prime}\left(x_{k}^{-}\right)\right)\left(u-u_{h}-v\right)\left(x_{k}\right)}_{\left(u_{h}^{\prime}\left(x_{k}^{+}\right)-u_{h}^{\prime}\left(x_{k}^{-}\right)\right)\left\langle\delta_{x-x_{k}}, u-u_{h}-v\right\rangle} \\
& +a(1)\left(u^{\prime}(1)-u_{h}^{\prime}(1)\right)\left(u-u_{h}-v\right)(1) \\
& -a(0)\left(u^{\prime}(0)-u_{h}^{\prime}(0)\right)\left(u-u_{h}-v\right)(0) \\
= & \int_{0}^{1}\left(a u_{h}^{\prime}\right)^{\prime}\left(u-u_{h}-v\right) \mathrm{d} x+\left(1-u_{h}^{\prime}(1)\right)\left(u(1)-u_{h}(1)-v(1)\right),
\end{aligned}
$$


for any $v \in V_{h}$ introduced by the Galerkin orthogonality. The terms $u_{h}^{\prime}\left(x_{k}^{-}\right)$and $u_{h}^{\prime}\left(x_{k}^{+}\right)$denote left and right limits, respectively, and $\left\langle\delta_{x-x_{k}}, u-u_{h}-v\right\rangle$ denotes the action of the Dirac delta distribution on the function $u-u_{h}-v$. This operation is well defined, since $u-u_{h}-v$ is continuous. The residual, viewed in the sense of distributions, has two parts. One part, $a u_{h}^{\prime \prime}$ and $1-u_{h}^{\prime}(1)$, consists of point masses at the nodal points, due to the jump of the piecewise constant derivative $u_{h}^{\prime}$, and the residual of the weak boundary condition, respectively. This part vanishes by choosing the test function $v=\pi\left(u-u_{h}\right)$ as the nodal interpolant of $u-u_{h}$, so that $u-u_{h}-v$ is zero in all nodal points. The remaining part of the residual $R:=a^{\prime} u_{h}^{\prime}$ does not vanish, and by evaluating the integral as the duality pairing $\int_{0}^{1} R\left(u-u_{h}-v\right) \mathrm{d} x=\left\langle R, u-u_{h}-v\right\rangle$ between the Sobolev spaces $\mathcal{H}^{-s}$ and $\mathcal{H}^{s}$ we obtain

$$
\left\|u-u_{h}\right\|_{E}^{2} \leq\|R\|_{-s}\left\|u-u_{h}-v\right\|_{s} .
$$

Then the standard a posteriori error estimate in the energy norm, $([6,7])$, is

$$
\left\|u-u_{h}\right\|_{E} \lesssim h^{1-s}\|R\|_{-s},
$$

since $\left\|u-u_{h}-\pi\left(u-u_{h}\right)\right\|_{s} \lesssim h^{1-s}\left\|u-u_{h}\right\|_{E}$ by (1.3). Since $R=a^{\prime} u_{h}^{\prime}=B^{\prime} e^{B} u_{h}^{\prime}$ has the regularity of white noise in space, we have $\|R\|_{L^{2}}=\infty$ for $s=0$. For other values of $s$, the negative Sobolev norm of the residual is not easily computed. Consequently, this direct application of the standard a posteriori error analysis does not provide an estimate that can be easily computed even for the preceding simple model problem.

This paper proposes computable, goal-oriented estimates for the Galerkin and quadrature errors committed in standard, continuous, piecewise linear finite element approximations of elliptic PDE with rough stochastic conductivities. A key insight for these estimates comes from studying the frequency content of the components of the Galerkin error functional, arising from the dual weighted residual analysis, for the simple model problem (1.1). In contrast to the case of smooth conductivities, we see that the components' high-frequency content, which cannot be computed directly, is non-negligible. Nevertheless, this high-frequency contribution can be approximated by low-frequency content. This formal frequency study suggests an assumption on scales that yields an estimator, based on local error indicators, for observables of the pathwise Galerkin error and hence the expected Galerkin error. Estimators for the expected quadrature error are also obtained under an assumption on scales where the stochastic cancellation effects present in the quadrature error are analyzed using tools from stochastic sensitivity analysis.

A posteriori estimates provide a quantitative measure of the quality of a numerical experiment. There is a rich literature concerning a posteriori error estimation for deterministic elliptic problems (see, for example, [23, 27, 9, 1]). While our error estimates rely on the computed solution to the finite element approximation and not on the analytic solution, we call our estimates "computable" as opposed to a posteriori as they require information about the regularity of the analytic solution. Here, this requisite information appears in the form of decay assumptions on the approximate solutions, which we call assumptions on scales. For the models with rough lognormal conductivity considered here, these decay rates are satisfied for all the linear observables under consideration as the given covariances are Lipschitz continuous. In principle our analysis can also treat conductivities with less regularity, such as conductivities whose covariances are not Lipschitz. In such cases, the decay assumptions would need to be obtained heuristically and might also depend on the particular choice 
of observable. We do not present a method for obtaining such rates through strictly a posteriori information. In spite of these technicalities, the estimates we propose are computable and can be useful when the standard a posteriori analysis fails and qualitative assessments based solely on a priori information might be misleading.

The proposed estimators could be used in constructing adaptive algorithms for variance reduction techniques for Monte Carlo (MC) methods. For variance reduction techniques, such as the Multilevel Monte Carlo (MLMC) method [26], Multi-Index Monte Carlo (MIMC) method [31], and Continuation MLMC (CMLMC) method [15], these novel, computable error estimates can be used to determine final level stopping criterion. An extension of the present theory would render it possible to also estimate mean square errors between numerical solutions on consecutive resolution levels. Since the mean square error implicitly bounds the variance, this may give rise to highly efficient a posteriori adaptive variance reduction methods for MLMC; see [33] for an application of such ideas in the setting of low regularity stochastic differential equations (SDE). Applications of these estimators to variance reduction and goal-oriented methods shall be the focus of the authors' future work.

The rest of this paper is organized as follows. In the next section, we clarify certain details of the model and recall results concerning the analysis of the finite element method. In $\S 3$, we present a formal frequency study of the Galerkin error functional, which shows that the high frequency content of the error, not observable on the computational grid, could, at least in principle, be approximated by the computable low frequency part under an assumption on a decay rate of Fourier modes. This observation motivates a similar derivation of a computable error estimate in $\S 4$, under a related assumption on scales. In particular, in $\S 4.2$, we give a computable estimate of the pathwise observable of the Galerkin error for the model problem (1.1) that relies on computations on only one computational mesh; that is, the computations are on one resolution level. We then present numerical experiments to test the proposed estimator for a two-dimensional problem in $\S 5$. Finally in $\S 6$, we show that another assumption on scales yields an estimate of the expected quadrature error committed in the finite element method.

2. The Model. We consider the isotropic diffusion problem

$$
-\operatorname{div}(a(\omega, x) \nabla u(\omega, x))=f(\omega, x)
$$

for $(\omega, x) \in \Omega \times \Gamma$ where $\Gamma$ is an bounded open subset of $\mathbf{R}^{d}$, for $d \geq 1$, and $(\Omega, \mathbb{F}, P)$ is a given probability space. To simplify our notation of the residual, we assume the homogeneous Dirichlet boundary condition $u(x)=u(\omega, x)=0$ for all $x \in \partial \Gamma P$-a.s. This equation arises in the geophysics literature in the study of time-independent groundwater flow on the local scale which is defined to be on the order of $10^{2}$ meters $([20,16,18])$. In this setting, equation (2.1) is Darcy's law with continuity where $a$ represents the $\log$ hydraulic conductivity, $f$ is a given source term, and the unknown $u$ represents the water pressure. A common feature of groundwater flow on the local scale is the spatial heterogeneity of the medium. In applications, prescribing $a$ precisely requires more information than is reasonably possible to acquire. This uncertainty in the problem data is thus incorporated by modeling $a$, and possibly $f$, as random fields. In applications to subsurface flow, the law of $a$ is typically assumed to be lognormal where the hydraulic conductivity, the Gaussian field $\log a$, has a Lipschitz covariance. For example, in two dimensions, one could consider the two-point isotropic covariance function given by $\operatorname{Cov}(x, y)=\sigma^{2} e^{-|x-y| / \ell}$, where $\sigma^{2}$ is the variance, $\ell$ the correlation length, and $|\cdot|$ is the Euclidean norm (see $[32,17,34]$ ). 
Another feature of groundwater flow on the local scale is that the correlation lengths involved are typically short; that is, the length scale for $\ell$ is significantly smaller than the scale of the problem domain but still too large for the application of stochastic homogenization techniques [14].

We begin by introducing the objects of interest to our finite element analysis. We study the variational form of equation (2.1) parameterized by $\omega \in \Omega$. That is, we seek $u \in \mathcal{H}^{1}(\Gamma)$ such that

$$
\int_{\Gamma} a(x) \nabla u(x) \cdot \nabla v(x) \mathrm{d} x=\int_{\Gamma} f(x) v(x) \mathrm{d} x \quad P \text {-a.s. }
$$

for all $v \in V=\mathcal{H}_{0}^{1}(\Gamma):=\left\{v \in \mathcal{H}^{1}(\Gamma): v(x)=0\right.$ for $x \in \partial \Gamma P$-a.s. in trace sense $\}$. Note that this formulation seeks a solution for almost all $\omega \in \Omega$. Let $V_{h}$ be the space of continuous piecewise linear functions on a mesh in $\Gamma$ vanishing along $\partial \Gamma$. The Galerkin formulation of (2.2) is: find $u_{h} \in V_{h}$ such that

$$
\int_{\Gamma} a(x) \nabla u_{h}(x) \cdot \nabla v_{h}(x) \mathrm{d} x=\int_{\Gamma} f(x) v_{h}(x) \mathrm{d} x \quad P \text {-a.s. }
$$

for all $v_{h} \in V_{h} \subset V$. In the finite element approximation of (2.3), a further error is committed by replacing the stiffness matrix components

$$
\int_{\Gamma} a(x) \nabla \phi_{i}(x) \cdot \nabla \phi_{j}(x) \mathrm{d} x
$$

with quadrature based ones, which are of the form

$$
\int_{\Gamma} \bar{a}(x) \nabla \phi_{i}(x) \cdot \nabla \phi_{j}(x) \mathrm{d} x
$$

so that the solution, including the contribution from the quadrature error, is given by $\bar{u}_{h} \in V_{h}$ such that

$$
\int_{\Gamma} \bar{a}(x) \nabla \bar{u}_{h}(x) \cdot \nabla v_{h}(x) \mathrm{d} x=\int_{\Gamma} \bar{f}(x) v_{h}(x) \mathrm{d} x \quad P \text {-a.s. }
$$

for all $v_{h} \in V_{h} \subset V$. As posed, (2.2), (2.3) and (2.4) are not uniformly elliptic with respect to $\omega$. Nevertheless, conditions under which unique solutions exist in the standard Bochner solution spaces, $L^{2}\left(\Omega, \mathbb{F}, \mathcal{H}_{0}^{1}\right)$, can be found, for example, in [12, 13], where the Fernique theorem [24] is used to overcome the lack of uniform ellipticity.

The analysis of finite element methods for elliptic PDE with sufficiently regular and uniformly positive and bounded stochastic coefficients is well established, see [19, $4,8,36,25,5,10]$. These analyses do not extend to model (2.1) as the conductivity is not guaranteed to be uniformly bounded and the regularity of the true solution is low. Instead, the two predominant strategies for approximating moments of observables are based on stochastic Galerkin methods and MC finite element methods.

Stochastic Galerkin methods rely on a truncated Karhunen-Loève expansion to obtain a reduced, or parametric, PDE that separates, in some sense, the problem's stochastic and deterministic components. Results in this direction have focused on questions of well-posedness, convergence rates, and a priori error estimates [28, 38, $12]$ and on adaptive schemes using the energy norm [29, 21, 22]. Due to the short correlation lengths involved in model (2.1), the reduced PDE typically results in a very 
high-dimensional stochastic parameter space that can be computationally limiting for calculating moments of observables.

An altogether different approach is to use MC methods. Such methods are favorable in high-dimensional situations since the convergence rates are dimensionally independent. We mention [14, 13, 40, 30], which analyze MLMC methods applied to various finite element methods for elliptic PDE with lognormal coefficients. In particular, [30] provides an analysis of mixed finite element methods that are useful for considering quantities of interest that depend on mass-conservative representation of the flux. All of these works focus on a priori estimates of the Galerkin finite element error.

In contrast, we give computable, goal-oriented, duality-based estimates for finite element methods in the MC framework. As discussed in the introduction, the expected error in the observable for the finite element approximation of (2.1) is given by

$$
\mathbf{E}\left[\left(g, u-\bar{u}_{h}\right)\right]=\mathbf{E}\left[\left(g, u-u_{h}\right)\right]+\mathbf{E}\left[\left(g, u_{h}-\bar{u}_{h}\right)\right]=: \mathbf{E} \mathcal{E}(g)+\mathbf{E} \mathcal{Q}(g),
$$

where $\mathbf{E} \mathcal{E}(g)$ and $\mathbf{E} \mathcal{Q}(g)$ are the expected Galerkin and quadrature errors, respectively, for the given observable. To be precise, we provide computable estimates for $\mathcal{E}(g)$, and hence also for $\mathbf{E} \mathcal{E}(g)$, and for $\mathbf{E} \mathcal{Q}(g)$ committed in a standard piecewise linear finite element approximation of (2.1). For deterministic problems with smooth conductivities, Johnson and his collaborators have long asserted the utility of such computable duality-based estimators (for example, see [23]).

We shall first examine the problem posed in the introduction of estimating $\mathcal{E}(g)$. Our estimates for error functionals of this form rely on local error indicators computed using the finite element approximation $u_{h}$ and the solution $\lambda$ to the following dual problem: find $\lambda \in V$ such that

$$
\int_{\Gamma} a(x) \nabla \lambda(x) \cdot \nabla v(x) \mathrm{d} x=\int_{\Gamma} g(x) v(x) \mathrm{d} x \quad P \text {-a.s. }
$$

for all $v \in V$. A simple calculation, using integration by parts and Galerkin orthogonality, shows that,

$$
\begin{aligned}
\mathcal{E}(g) & =\left(u-u_{h},-\nabla \cdot(a \nabla \lambda)\right) \\
& =\left(a \nabla\left(u-u_{h}\right), \nabla \lambda\right) \\
& =\left(a \nabla\left(u-u_{h}\right), \nabla\left(\lambda-v_{h}\right)\right) \\
& =\left(f, \lambda-v_{h}\right)-\left(a \nabla u_{h}, \nabla\left(\lambda-v_{h}\right)\right) \\
& =\int_{\Gamma}\left(f+\nabla \cdot\left(a \nabla u_{h}\right)\right)\left(\lambda-v_{h}\right) \mathrm{d} x
\end{aligned}
$$

for any $v_{h} \in V_{h}$, in the sense of distributions. This is a similar calculation as in equation (1.4). The error based on the residual is therefore, in distribution form,

$$
\mathcal{E}(g)=\int_{\Gamma} R(\omega, x)\left(\lambda(\omega, x)-v_{h}(x)\right) \mathrm{d} x=\left\langle R, \lambda-v_{h}\right\rangle,
$$

for any $v_{h} \in V_{h}$, where the residual $R:=f+\nabla \cdot\left(a \nabla u_{h}\right)$ is a distribution containing also the jump terms at the edges since the residual is a measure on the edges and not a function. For problem (1.1), this quantity reduces to

$$
\mathcal{E}(g)=\int_{0}^{1}\left(a u_{h}^{\prime}\right)^{\prime}\left(\lambda-v_{h}\right) \mathrm{d} x+\left(1-u_{h}(1)\right)\left(\lambda(1)-v_{h}(1)\right),
$$


as shown in (1.4), and the remaining part of the residual, that does not vanish, is $R=a^{\prime} u_{h}^{\prime}$. A formal analysis of the frequency content of (2.8) offers further insight into suitable local error indicators.

3. Numerical Study of the Frequency Content. Next, we undertake a formal study of the frequency content of the residual error (2.8) for the simple model problem (1.1). The error contains high-frequency content that is non-negligible. Under assumptions on the decay of the frequency content, we observe that the highfrequency contribution in the error observable can be estimated in terms of the lowfrequency content. Although computations are provided only for a one-dimensional example, this frequency study provides insight into why the standard a posteriori analysis fails. Motivated by the observations in this section, we derive error estimators in the subsequent sections through an analysis of scales and give a computable estimator for (1.1) requiring only one level of the mesh size in $\S 4.2$.

From equation (2.8), we express the remaining part of the residual and the dual terms in Fourier series,

$$
R(x)=\sum_{n=-\infty}^{\infty} r_{n} e^{2 \pi i n x} \quad \text { and } \quad\left(\lambda-v_{h}\right)(x)=\sum_{n=-\infty}^{\infty} \lambda_{n} e^{2 \pi i n x},
$$

where $r_{n}$ and $\lambda_{n}$ denote the Fourier coefficients and where we are free to choose $v_{h} \in V_{h}$ to ensure a continuous periodic extension of $\lambda-v_{h}$. In particular, we choose $v_{h}=\pi_{h} \lambda$, where $\pi_{h}: V \rightarrow V_{h}$ is the nodal interpolant. Naively choosing $v_{h}=0$ would result in a decay rate for $\lambda_{n}$ of at most $O\left(n^{-1}\right)$, which is non-optimal due to the jump discontinuity introduced by the particular choice of boundary conditions for the model problem. Further, we shall split $\lambda-v_{h}$ into low- and high-frequency components,

$$
\lambda-v_{h}=\sum_{|n|<n^{\star}} \lambda_{n} e^{2 \pi i n x}+\sum_{|n| \geq n^{\star}} \lambda_{n} e^{2 \pi i n x}=: \underline{\lambda}+\bar{\lambda},
$$

for $n^{\star}=C / h$, where $C=C(\omega)$ is a constant independent of $h$. The mesh size, $h$, limits the frequencies obtained in the computed finite element solution, $u_{h}$. In this sense, $\underline{\lambda}$ and $\bar{\lambda}$, respectively, represent the low- and high-frequency components relative to the residual that is computed using the finite element solution, $u_{h} \cdot \bar{\lambda}$ cannot be approximated based on $V_{h}$ or $V_{\frac{h}{2}}$ but $\underline{\lambda}$ can be approximated based on $V_{h}$ and $V_{\frac{h}{2}}$.

In Figure 1, an estimate of

$$
|\mathcal{E}(1)|:=\left|\sum_{n=-\infty}^{\infty} r_{n} \lambda_{n}\right|,
$$

the Galerkin error, and

$$
\left|\mathcal{E}_{L}(1)\right|:=\left|\sum_{|n|<n^{\star}} r_{n} \lambda_{n}\right|
$$

the low-frequency component of the Galerkin error, for the observable $g=1$, are plotted for a smooth conductivity, $a(x)=x+1$, and for one realization of a rough lognormal conductivity, $a(x)=e^{B(x)}$, where the field $a$ has covariance function,

$$
\operatorname{Cov}(a(x), a(y))=e^{\frac{1}{2}(x(1-x)+y(1-y))}\left(e^{\min (x, y)-x y}-1\right) .
$$



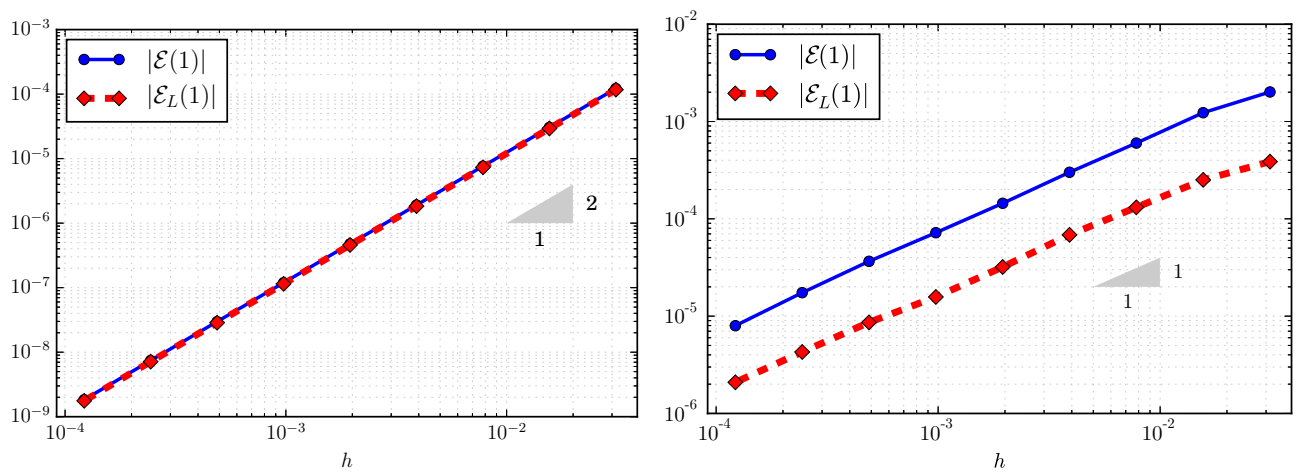

Figure 1: The high-frequency content contributes to the Galerkin error for one realization of the rough lognormal conductivity, $a(x)=e^{B(x)}$, (right-hand panel) in contrast to a smooth conductivity, $a(x)=1+x$, (left-hand panel) where the contribution is negligible.

Here the Fourier coefficients are given by the discrete Fourier transform, $v_{h}=\pi_{h} \lambda$ is chosen, $\lambda$ is computed on a reference mesh of size $h=2^{-16}$, and all the finite element calculations use a quadrature mesh of size $k=2^{-23}$ to avoid polluting the Galerkin error with quadrature error. We observe that in the case of the smooth $a$, the contribution to the error from the high-frequency component is expected to be negligible, as is indicated in the left-hand panel. In the case of the rough lognormal $a$, see the right-hand panel, the low-frequency component does not sufficiently capture the Galerkin error indicating a non-negligible contribution from $\bar{\lambda}$. Although the high frequency component, which requires a space much finer than $V_{h}$ to be resolved, contributes to the error in this setting, we are able to estimate the contribution from $\bar{\lambda}$ in terms of $\underline{\lambda}$ if an assumption on the decay of the Fourier coefficients is made.

Remark 1. Figure 1 also demonstrates that the Galerkin error in the piecewise linear finite element approximation of (1.1) is on the order of $O(h)$ in the case of the rough lognormal conductivity as compared to $O\left(h^{2}\right)$ for the smooth conductivity.

If we assume that

$$
\left|r_{n} \lambda_{n}\right|+\left|r_{-n} \lambda_{-n}\right| \leq C n^{-2 \alpha},
$$

for positive $n$ and a positive $C=C(\omega)$, then

$$
\mathcal{E}(g) \leq \tilde{C} h^{2 \alpha-1}
$$

for $\alpha \in(1 / 2,3 / 2)$ for a random variable $\tilde{C}=\tilde{C}(\omega)$ not depending on $h$. This estimate is obtained by first applying the splitting (3.1) to (2.8) and then by using the decay assumption (3.2).

To wit, the frequency splitting yields

$$
\mathcal{E}(g)=\int_{0}^{1} R(x)\left(\underline{\lambda}-\pi_{h} \underline{\lambda}\right)(x) \mathrm{d} x+\int_{0}^{1} R(x) \bar{\lambda}(x) \mathrm{d} x .
$$

Applying $\underline{\lambda}-\pi_{h} \underline{\lambda} \simeq C_{0} h^{2} \underline{\lambda}^{\prime \prime}$, a standard localization estimate with deterministic 
interpolation constant $C_{0}$, the error can then be approximated by

$$
|\mathcal{E}(g)| \leq \sum_{|n|<n^{\star}} C_{0} h^{2} n^{2}\left|r_{n}\right|\left|\lambda_{n}\right|+\sum_{|n| \geq n^{\star}}\left|r_{n} \lambda_{n}\right|
$$

Then, we have

$$
|\mathcal{E}(g)| \leq \sum_{|n|<C / h} C_{0} h^{2} \frac{n^{2}}{n^{2 \alpha}}+\sum_{|n| \geq C / h} \frac{C}{n^{2 \alpha}}
$$

by the decay assumption (3.2). The high-frequency contribution, arising from the latter sum, can be estimated by

$$
2 \int_{C / h}^{\infty} s^{-2 \alpha} \mathrm{d} s=2\left[\frac{-s^{-2 \alpha+1}}{1-2 \alpha}\right]_{C / h}^{\infty}=\frac{2}{2 \alpha-1}\left(\frac{h}{C}\right)^{2 \alpha-1}
$$

provided $\alpha>1 / 2$, and the low-frequency contribution, arising from the first sum, by

$$
2 C_{0} h^{2} \int_{0}^{C / h} s^{2-2 \alpha} \mathrm{d} s=\frac{2 C_{0}}{3-2 \alpha} C^{3-2 \alpha} h^{2 \alpha-1}
$$

provided $\alpha<3 / 2$.

Thus, we have the estimate

$$
|\mathcal{E}(g)| \leq 2\left(\frac{C_{0} C^{3-2 \alpha}}{3-2 \alpha}+\frac{C^{1-2 \alpha}}{2 \alpha-1}\right) h^{2 \alpha-1},
$$

for $\alpha \in(1 / 2,3 / 2)$. In contrast, for a smooth conductivity $a$, we have

$$
|\mathcal{E}(g)| \leq 2\left(\frac{C_{0}}{2 \alpha-3} h^{2}+\frac{1}{2 \alpha-1} h^{2 \alpha-1}\right)
$$

for $\alpha>3 / 2$, where the contribution from the high frequencies is clearly negligible. While the high frequency error contribution for the rough lognormal conductivity cannot be observed on the computational grid, the foregoing argument shows that it is of the same order, $h^{2 \alpha-1}$, as the low frequency error that is possible to detect in a numerical simulation. Therefore, a naive approach that only takes into account the smooth part of the error would always be a factor wrong.

Returning to consider the validity of our initial assumption, we see that (3.2) is satisfied for a constant $\alpha$ in the case of the simple model problem (1.1). Recall that the residual has the form $R(x)=a^{\prime} u_{h}^{\prime}=B^{\prime} e^{B} u_{h}^{\prime} \approx W^{\prime} e^{B} u_{h}^{\prime}$, where $W^{\prime}$ is a white noise in space. The white noise has a power spectrum density that is flat, that is, $\left|\mathcal{F}_{n}\left(W^{\prime}\right)\right|^{2}=O_{P}(1)$, that is, $O(1) P$-a.s., where $\mathcal{F}_{n}(f)$ denotes the $n$th Fourier mode of $f(x)$. Hence $\left|\mathcal{F}_{n}(R)\right|=O_{P}(1)$. For the dual, we have the explicit representation $\lambda^{\prime}=G / a$, where $G$ is the primitive of $g$. If we suppose $G=1$ and approximate $1 / a \approx 1-B \approx 1-W$, then

$$
\left|\mathcal{F}_{n}(\lambda)\right|=O_{P}\left(n^{-1}\right)\left|\mathcal{F}_{n}\left(\lambda^{\prime}\right)\right|=O_{P}\left(n^{-1}\right)\left|\mathcal{F}_{n}(W)\right|=O_{P}\left(n^{-2}\right) .
$$

Assuming more generally that $g \in C^{0}([0,1])$, we have by Parseval's identity that $\|g\|_{2}=\sum_{k}\left|\mathcal{F}_{k}(g)\right|^{2}<\infty$, so we may assume $\mathcal{F}_{n}(g) \rightarrow 0$ as $n \rightarrow \infty$. Then we obtain 
the same rate,

$$
\begin{aligned}
\left|\mathcal{F}_{n}(\lambda)\right| & =O_{P}\left(n^{-1}\right)\left|\mathcal{F}_{n}\left(\lambda^{\prime}\right)\right| \\
& =O_{P}\left(n^{-1}\right)\left|\mathcal{F}_{n}(G(1-W))\right| \\
& =O_{P}\left(n^{-2}\right)\left(\left|\mathcal{F}_{n}(g)\right|+\left|\mathcal{F}_{n}\left(g W+G W^{\prime}\right)\right|\right)=O_{P}\left(n^{-2}\right) .
\end{aligned}
$$

Here we have used the convolution rule for Fourier series, Parseval's identity, and Hölder's inequality to conclude that

$$
\mathcal{F}_{n}(g W)=(\mathcal{F}(g) * \mathcal{F}(W))_{n}=\sum_{k} \mathcal{F}_{k}(g) \mathcal{F}_{n-k}(W) \leq\|g\|_{2}\|W\|_{2}=O_{P}(1),
$$

since $W$ is in $C^{0}([0,1])$ and bounded, and thus $W \in L^{2}(0,1) P$-a.s. Computing the integral of $\mathcal{F}_{n}\left(G W^{\prime}\right)$ is slightly more cumbersome, but once again one can use that $\|W\|_{2}=O_{P}(1)$, neglecting constants, write the integral with $W^{\prime}$ expanded in the Karhunen-Loève basis to see that

$$
\begin{aligned}
\mathcal{F}_{n}\left(G W^{\prime}\right) & =\sum_{k} \mathcal{F}_{n+k}(G) \mathcal{F}_{k}\left(W^{\prime}\right) \\
& =\sum_{k} \frac{\mathcal{F}_{n+k}(g)}{|n+k|+1} \mathcal{F}_{k}\left(W^{\prime}\right) \\
& =\sum_{k} \mathcal{F}_{n+k}(g) \mathcal{F}_{k}\left(\frac{W^{\prime}}{1+|k|}\right) \\
& =\sum_{k} \mathcal{F}_{n+k}(g) \mathcal{F}_{k}(W) \\
& \leq\|g\|_{2}\|W\|_{2}=O_{P}(1) .
\end{aligned}
$$

Thus, the decay rate is satisfied with $\alpha=1$ in the case of the simple model problem when $g \in C^{0}([0,1])$.

This rate is also observed experimentally for $g=1$ as well as $g=\delta_{\frac{1}{2}}$. Using the explicit representations available in the one-dimensional model problem, the product of the Fourier coefficients corresponding to $\mathcal{E}(1)$, and also for $\mathcal{E}\left(\delta_{\frac{1}{2}}\right)$, are plotted in Figure 2, where $\delta_{\frac{1}{2}}(x):=\delta(x-1 / 2)$ is the $\delta$-function centered at $x=1 / 2$. Here the computations are for one sample path of the log-Brownian bridge process, $a=e^{B}$, for elements of size $h=2^{-10}$, with a quadrature mesh of size $k=2^{-25}$, and the decay rate is fitted using the method of least squares. The Fourier content decays at a constant rate, corresponding to $\alpha \approx 1$, for both the observables $g=1$ and $g=\delta_{\frac{1}{2}}$. In particular, for constant $\alpha=1$, the contributions from the high- and low-frequency components are $2 C^{-1}$ and $2 C_{0} C$, respectively, and therefore

$$
\frac{\text { low }}{\text { total }}=\frac{\text { low }}{\text { low }+ \text { high }}=\frac{1}{1+\frac{1}{C_{0} C^{2}}}
$$

is the ratio of the contribution of the low-frequency part to the total frequency content of the error.

With the insight obtained from the formal analysis in the present section, we anticipate that only a fraction of the total error can be found on the computational grid used. If this fraction would be known, then the total error could be estimated as an error estimator from the computational grid divided by that fraction. In the 

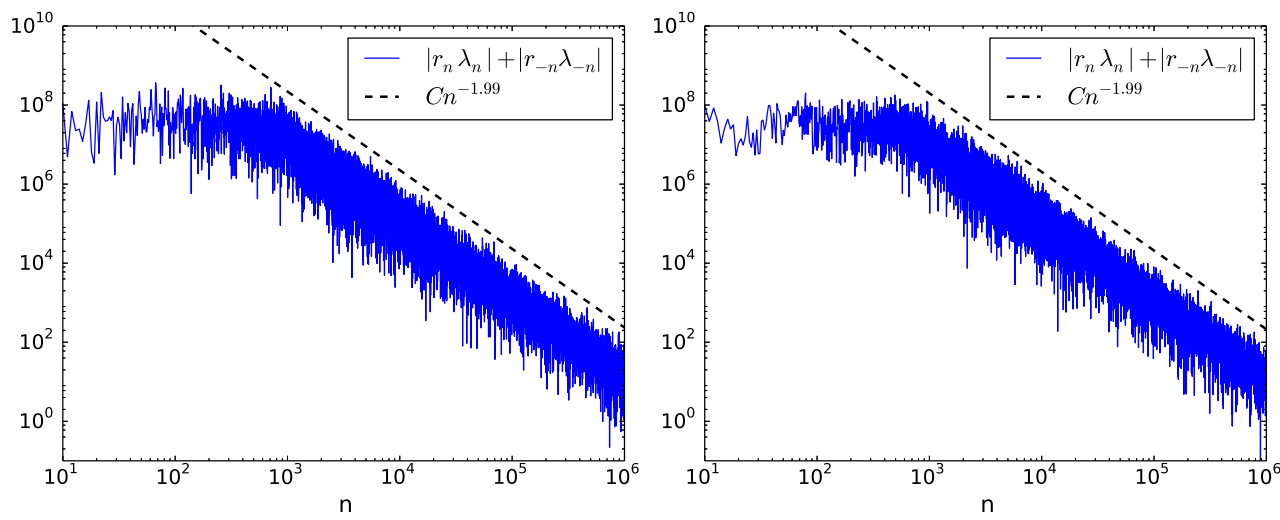

Figure 2: Pathwise, the Fourier content decays at a constant rate, observed to be $O\left(n^{-1.99}\right)$, for the observables $g=1$ (left-hand panel) and $g=\delta_{\frac{1}{2}}$ (right-hand panel).

next section we show rigorously that frequency splitting can be achieved under an assumption on scales related to (3.2). The analysis given there also gives the factor that should multiply the "observable" error contribution from a computational grid.

\section{Analysis of scales.}

4.1. Frequency splitting via an assumption on scales. Presently we derive the error estimators via a general telescoping argument in which the frequency splitting required to drive our analysis is achieved by an assumption on scales. This analysis yields a similar estimate as the frequency study in the previous section for the one-dimensional model problem and can be readily applied to higher-dimensional problems. We will need the solutions $\lambda_{h}$ to the discretized version of the dual equation (2.6), given by

$$
\int_{\Gamma} a(x) \nabla \lambda_{h}(x) \cdot \nabla v_{h}(x) \mathrm{d} x=\int_{\Gamma} g(x) v_{h}(x) \mathrm{d} x \quad P \text {-a.s. }
$$

for all $v_{h} \in V_{h} \subset V$.

THEOREM 4.1. Assume that, for positive constants $\alpha$ and $\beta$, and for a positive random variable $C=C(\omega)$, the discrete solutions $u_{h}$ and $\lambda_{h}$ to the equations (2.3) and (4.1) satisfy

$$
\int_{\Gamma} a(x)\left|\nabla\left(u_{h / 2}-u_{h}\right)(x) \cdot \nabla\left(\lambda_{H / 2}-\lambda_{H}\right)(x)\right| \mathrm{d} x \leq C h^{\alpha} H^{\beta}
$$

for sufficiently small mesh sizes $h$ and $H$. Then the Galerkin error $\mathcal{E}(g)$, defined in (2.5), satisfies

$$
|\mathcal{E}(g)| \leq \frac{C h^{\alpha+\beta}}{\left(1-2^{-\alpha}\right)\left(1-2^{-\beta}\right)}
$$

Proof. Letting $v_{h}=\lambda_{h}$, we rewrite the Galerkin error (2.7) as

$$
\mathcal{E}(g)=\int_{\Gamma} a(x) \nabla\left(u-u_{h}\right)(x) \cdot \nabla\left(\lambda-\lambda_{h}\right)(x) \mathrm{d} x .
$$


Expanding the quantities containing differences in the primal and dual variables in telescoping sums in $m$ and $n$, we obtain

$$
\begin{aligned}
|\mathcal{E}(g)| & =\left|\sum_{m, n=0}^{\infty} \int_{\Gamma} a(x) \nabla\left(u_{h / 2^{m+1}}-u_{h / 2^{m}}\right)(x) \cdot \nabla\left(\lambda_{h / 2^{n+1}}-\lambda_{h / 2^{n}}\right)(x) \mathrm{d} x\right| \\
& \leq \sum_{m, n=0}^{\infty} \int_{\Gamma} a(x)\left|\nabla\left(u_{h / 2^{m+1}}-u_{h / 2^{m}}\right)(x) \cdot \nabla\left(\lambda_{h / 2^{n+1}}-\lambda_{h / 2^{n}}\right)(x)\right| \mathrm{d} x \\
& \leq \sum_{m, n=0}^{\infty} C h^{\alpha} h^{\beta} 2^{-m \alpha} 2^{-n \beta}=\frac{C h^{\alpha+\beta}}{\left(1-2^{-\alpha}\right)\left(1-2^{-\beta}\right)} \cdot
\end{aligned}
$$

One can estimate $\mathcal{E}(g)$ by computing

$$
\tilde{F}(h):=\int_{\Gamma} a \nabla\left(u_{h / 2}-u_{h}\right) \cdot \nabla\left(\lambda_{h / 2}-\lambda_{h}\right) \mathrm{d} x \approx C^{\prime}(\omega) h^{\alpha+\beta},
$$

a quantity of the same order as $\mathcal{E}(g)$, for a random variable $C^{\prime}$ independent of $h$, which relies on local error indicators on two levels, that is, on two nested computational meshes. In practice, we shall estimate $\mathcal{E}(g)$ by

$$
F(h):=\int_{\Gamma} a(x)\left|\nabla\left(u_{h / 2}-u_{h}\right)(x) \cdot \nabla\left(\lambda_{h / 2}-\lambda_{h}\right)(x)\right| \mathrm{d} x,
$$

for the reasons outlined below.

For some observables, the Galerkin error may be small due to non-stochastic cancellations in the error indicators. In Figures 3 and 4, we plot, element-wise, the local error indicators for several observables for the one-dimensional model problem to illustrate the possible effects of cancellations. That is, we split the domain of the integral in $\tilde{F}(h)$ over each $h$-element and plot the resulting element-wise contributions to the Galerkin error corresponding to $g=1$ and $g=\delta_{\frac{1}{2}}$ in Figure 3 and $g=\cos (2 \pi \cdot)$ in Figure 4. These error indicators are computed for $h=2^{-9}$ using a quadrature mesh, $k=2^{-22}$. In contrast to the indicators corresponding to $g=\cos (2 \pi \cdot)$, displayed in Figure 4, we observe that the error indicators corresponding to $g=1$ and $g=\delta_{\frac{1}{2}}$ will not exhibit extensive cancellations when the error estimator is computed.

Trivially, the estimator may vanish due to cancellations of the indicators, for a particular $h$ or for a particular choice of observable. However, it is not easy to say when such cancellations occur, or how to take advantage of them in order to make better predictions of the size of the error. Furthermore, if we require that the signed estimator $F(h)$ is bounded away from zero, we obtain the following corollary to Theorem 4.1:

Corollary 4.2. If the estimator $F(h)$, defined in (4.3), satisfies

$$
c \leq \frac{F(h)}{h^{\alpha+\beta}} \leq C
$$

for positive constants $c=c(\omega)$ and $C=C(\omega)$, both independent of $h$, the Galerkin error $\mathcal{E}(g)$, defined in (2.5), satisfies

$$
|\mathcal{E}(g)| \leq \frac{C F(h)}{c\left(1-2^{-\alpha}\right)\left(1-2^{-\beta}\right)} .
$$



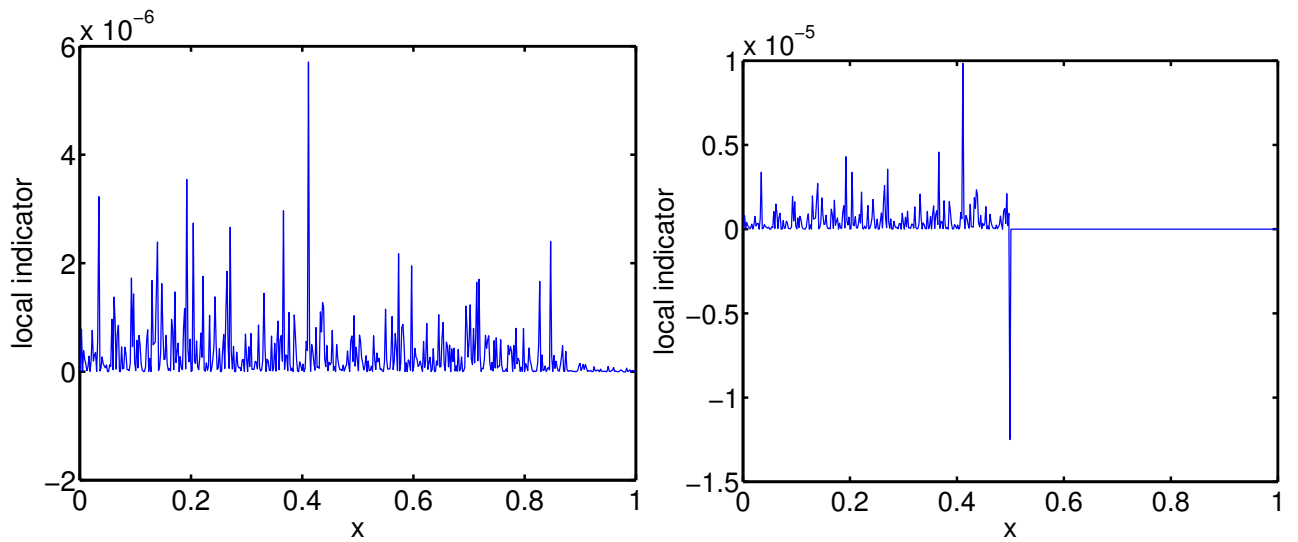

Figure 3: The local error indicators for one realization of the error committed in approximating two generic observables, $g=1$ (left-hand panel) and $g=\delta_{\frac{1}{2}}$ (righthand panel), do not sum to zero.

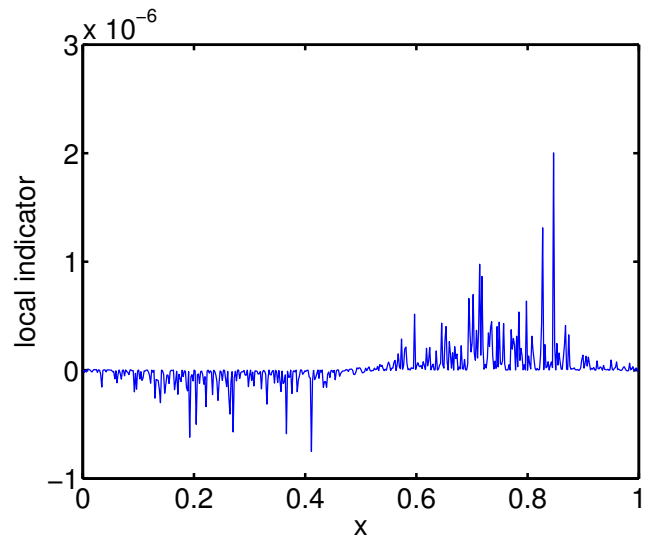

Figure 4: The local error indicators for one realization of the error committed in approximating a pathological observable, $g=\cos (2 \pi \cdot)$, specially constructed to exhibit extensive cancellations in the estimator.

Next, we derive a computable estimate for the one-dimensional model problem equation (1.1). Then, in the remainder of this section, we determine the factor in the above estimate and provide some experiments testing our estimator for the model problem.

4.2. Computable error for the simple model problem. In Theorem 4.4 below, we give a computable estimator, based on local error indicators, for the simple model problem (1.1). This estimator relies on a representation of the discrete primal and dual solutions, given in Lemma 4.3, where the value of $a$ in the variational formulation is averaged over the $h$-elements. That is, let $a_{h}$ be the spatial average of $a$ 
over the $h$-elements,

$$
a_{h}(x)=\sum_{K_{h}} \mathbf{1}_{K_{h}}(x) \frac{1}{\left|K_{h}\right|} \int_{K_{h}} a(s) \mathrm{d} s,
$$

for all $h$-elements $K_{h}$, where

$$
\mathbf{1}_{K_{h}}(x)= \begin{cases}1 & x \in K_{h} \\ 0 & x \notin K_{h}\end{cases}
$$

and where $\left|K_{h}\right|=\int_{K_{h}} \mathrm{~d} x$ denotes the size of element $K_{h}$. The finite element solution $u_{h}$ to (1.1) satisfies the discrete variational equation

$$
\int_{0}^{1} a u_{h}^{\prime} v_{h}^{\prime} \mathrm{d} x=v_{h}(1) \quad P \text {-a.s. }
$$

for all $v_{h} \in V_{h}$, where $V_{h}$ is the space of all piecewise linear functions $v_{h}$ on $(0,1)$ with grid spacing $h$ that satisfy $v_{h}(0)=0$. Since $u_{h}^{\prime}$ and $v_{h}^{\prime}$ are constant on each $h$-element we have

$$
\int_{0}^{1} a u_{h}^{\prime} v_{h}^{\prime} \mathrm{d} x=\int_{0}^{1} a_{h} u_{h}^{\prime} v_{h}^{\prime} \mathrm{d} x .
$$

Likewise, the discrete dual, $\lambda_{h}$, is the solution of the variational equation

$$
\int_{0}^{1} a \lambda_{h}^{\prime} v_{h}^{\prime} \mathrm{d} x=\int_{0}^{1} a_{h} \lambda_{h}^{\prime} v_{h}^{\prime} \mathrm{d} x=\int_{0}^{1} g v_{h} \mathrm{~d} x
$$

for all piecewise linear test functions, $v_{h}$. For the observable $g$, let

$$
G(x)=-\int_{x}^{1} g(s) \mathrm{d} s
$$

denote the primitive function of the observable $g$. We then have the following representations for the derivatives of the discrete primal and dual solutions.

Lemma 4.3. The derivatives of the discrete primal and dual solutions $u_{h}$ and $\lambda_{h}$ are given by

$$
\begin{aligned}
u_{h}^{\prime} & =1 / a_{h}, \\
\lambda_{h}^{\prime} & =G_{h} / a_{h},
\end{aligned}
$$

where

$$
G_{h}(x)=\sum_{K_{h}} \mathbf{1}_{K_{h}}(x) \frac{1}{\left|K_{h}\right|} \int_{K_{h}} G(s) \mathrm{d} s .
$$

Proof. The expression for $u_{h}$ follows directly from (4.6) and (4.7). The expression for $\lambda_{h}$ follows by integration by parts of the right-hand side in (4.8):

$$
\int_{0}^{1} a_{h} \lambda_{h}^{\prime} v_{h}^{\prime} \mathrm{d} x=\int_{0}^{1} G v_{h}^{\prime} \mathrm{d} x=\int_{0}^{1} G_{h} v_{h}^{\prime} \mathrm{d} x,
$$

where we use $v_{h}(0)=G(1)=0$. Since this equation must hold for all test functions, $v_{h}$, the expression for $\lambda_{h}$ in the lemma follows. 
Each interval in the grid with step length $h$ corresponds to precisely two subintervals in the grid with length $h / 2$. In order to have concise notation, entities corresponding to the left of these two intervals are marked with a minus (-) sign, and entities corresponding to the right subinterval are marked with a plus $(+)$ sign. The following somewhat surprisingly simple equality gives a computable estimator for the observable for the Galerkin error for the simple model problem (1.1) based on only one mesh size.

THEOREM 4.4.

$$
\tilde{F}(h)=\int_{0}^{1} a\left(u_{h / 2}-u_{h}\right)^{\prime}\left(\lambda_{h / 2}-\lambda_{h}\right)^{\prime} \mathrm{d} x=\sum_{K_{h}} \frac{h^{3}}{16} a_{h}^{*} D^{2} u_{h / 2} D^{2} \lambda_{h / 2},
$$

where the sum is over all h-elements in the coarse mesh, $K_{h}$, and $a_{h}^{*}$ is the harmonic mean of $a_{h / 2}$ over each $K_{h}$, i.e.,

$$
a_{h}^{*}=\frac{2}{\frac{1}{a_{h / 2}^{+}}+\frac{1}{a_{h / 2}^{-}}} .
$$

The second-order difference in each coarse grid interval is given by

$$
D^{2} u_{h / 2}:=\frac{D u_{h / 2}^{+}-D u_{h / 2}^{-}}{h / 2}=\frac{\left(u_{h / 2}^{+}\right)^{\prime}-\left(u_{h / 2}^{-}\right)^{\prime}}{h / 2}
$$

and similarly for $\lambda_{h / 2}$.

Proof. By Lemma 4.3, we have

$$
D^{2} u_{h / 2}=D\left(1 / a_{h / 2}\right)=\frac{2}{h}\left(\frac{1}{a_{h / 2}^{+}}-\frac{1}{a_{h / 2}^{-}}\right)=\frac{2}{h} \frac{a_{h / 2}^{-}-a_{h / 2}^{+}}{a_{h / 2}^{+} a_{h / 2}^{-}} .
$$

We want to compare this with the difference between first-order derivatives on grids with lengths $h / 2$ and $h$,

$$
\left(u_{h / 2}^{ \pm}\right)^{\prime}-u_{h}^{\prime}=\frac{1}{a_{h / 2}^{ \pm}}-\frac{1}{a_{h}}=\frac{a_{h}-a_{h / 2}^{ \pm}}{a_{h} a_{h / 2}^{ \pm}}= \pm \frac{a_{h / 2}^{-}-a_{h / 2}^{+}}{2 a_{h} a_{h / 2}^{ \pm}},
$$

where in the last equality we used

$$
a_{h}=\frac{a_{h / 2}^{+}+a_{h / 2}^{-}}{2}
$$

Combining (4.11) and (4.12), we obtain

$$
\left(u_{h / 2}^{ \pm}\right)^{\prime}-u_{h}^{\prime}= \pm \frac{h}{2} \frac{a_{h / 2}^{+} a_{h / 2}^{-}}{2 a_{h} a_{h / 2}^{ \pm}} D^{2} u_{h / 2}= \pm h \frac{a_{h / 2}^{\mp}}{4 a_{h}} D^{2} u_{h / 2} .
$$

Proceeding in a similar manner, we now prove that $\lambda_{h}$ and $\lambda_{h / 2}$ satisfy the same relation, i.e.,

$$
\left(\lambda_{h / 2}^{ \pm}\right)^{\prime}-\lambda_{h}^{\prime}= \pm h \frac{a_{h / 2}^{\mp}}{4 a_{h}} D^{2} \lambda_{h / 2} .
$$


We begin by computing $D^{2} \lambda_{h / 2}$ using Lemma 4.3. This yields

$$
\begin{aligned}
D^{2} \lambda_{h / 2}=D\left(\frac{G_{h / 2}}{a_{h / 2}}\right) & =\frac{2}{h}\left(\frac{G_{h / 2}^{+}}{a_{h / 2}^{+}}-\frac{G_{h / 2}^{-}}{a_{h / 2}^{-}}\right) \\
& =\frac{2}{h}\left(\frac{G_{h}}{a_{h / 2}^{+}}-\frac{G_{h}}{a_{h / 2}^{-}}+\frac{G_{h / 2}^{+}-G_{h}}{a_{h / 2}^{+}}+\frac{G_{h}-G_{h / 2}^{-}}{a_{h / 2}^{-}}\right)
\end{aligned}
$$

and, since $G_{h / 2}^{+}-G_{h}=G_{h}-G_{h / 2}^{-}=\left(G_{h / 2}^{+}-G_{h / 2}^{-}\right) / 2$, we obtain

$$
D^{2} \lambda_{h / 2}=\frac{2}{h}\left(G_{h} \frac{a_{h / 2}^{-}-a_{h / 2}^{+}}{a_{h / 2}^{+} a_{h / 2}^{-}}+\frac{G_{h / 2}^{+}-G_{h / 2}^{-}}{a_{h}^{*}}\right)
$$

where we recall, for the convenience of the reader, that

$$
\frac{1}{a_{h}^{*}}=\frac{1}{2}\left(\frac{1}{a_{h / 2}^{+}}+\frac{1}{a_{h / 2}^{-}}\right) .
$$

The difference between first-order derivatives on grids with lengths $h / 2$ and $h$ is then given by

$$
\begin{aligned}
\left(\lambda_{h / 2}^{ \pm}\right)^{\prime}-\lambda_{h}^{\prime}=\frac{G_{h / 2}^{ \pm}}{a_{h / 2}^{ \pm}}-\frac{G_{h}}{a_{h}} & =\frac{G_{h / 2}^{ \pm}-G_{h}}{a_{h / 2}^{ \pm}}+G_{h}\left(\frac{1}{a_{h / 2}^{ \pm}}-\frac{1}{a_{h}}\right) \\
& =\frac{G_{h / 2}^{ \pm}-G_{h}}{a_{h / 2}^{ \pm}} \pm G_{h} \frac{a_{h / 2}^{-}-a_{h / 2}^{+}}{2 a_{h} a_{h / 2}^{ \pm}}
\end{aligned}
$$

Combining equations (4.15) and (4.16) yields (4.14):

$$
\begin{aligned}
& \left(\lambda_{h / 2}^{ \pm}\right)^{\prime}-\lambda_{h}^{\prime} \mp h \frac{a_{h / 2}^{\mp}}{4 a_{h}} D^{2} \lambda_{h / 2} \\
& =\frac{G_{h / 2}^{ \pm}-G_{h}}{a_{h / 2}^{ \pm}} \pm G_{h} \frac{a_{h / 2}^{-}-a_{h / 2}^{+}}{2 a_{h} a_{h / 2}^{ \pm}} \mp h \frac{a_{h / 2}^{\mp}}{4 a_{h}} \frac{2}{h}\left(G_{h} \frac{a_{h / 2}^{-}-a_{h / 2}^{+}}{a_{h / 2}^{+} a_{h / 2}^{-}}+\frac{G_{h / 2}^{+}-G_{h / 2}^{-}}{a_{h}^{*}}\right) \\
& =\frac{G_{h / 2}^{ \pm}-G_{h}}{a_{h / 2}^{ \pm}} \mp \frac{a_{h / 2}^{\mp}}{2 a_{h} a_{h}^{*}}\left(G_{h / 2}^{+}-G_{h / 2}^{-}\right) \\
& = \pm \frac{G_{h / 2}^{+}-G_{h / 2}^{-}}{2}\left(\frac{1}{a_{h / 2}^{ \pm}}-\frac{a_{h / 2}^{\mp}}{a_{h} a_{h}^{*}}\right)=0,
\end{aligned}
$$

where in the last equality we used

$$
a_{h} a_{h}^{*}=\frac{a_{h / 2}^{+}+a_{h / 2}^{-}}{\frac{1}{a_{h / 2}^{+}}+\frac{1}{a_{h / 2}^{-}}}=a_{h / 2}^{+} a_{h / 2}^{-} .
$$

Finally, combining (4.13) and (4.14), the expressions for the differences of firstorder derivatives on the nested grids for the primal and dual variables, we obtain

$$
a_{h / 2}^{ \pm}\left(D u_{h / 2}^{ \pm}-D u_{h}\right)\left(D \lambda_{h / 2}^{ \pm}-D \lambda_{h}\right)=\left(\frac{h}{4}\right)^{2} \frac{a_{h / 2}^{ \pm}\left(a_{h / 2}^{\mp}\right)^{2}}{a_{h}^{2}} D^{2} u_{h / 2} D^{2} \lambda_{h / 2},
$$


an expression for the integrand in the statement of the theorem. By summing over $K_{h}$, the coarse grid with step length $h$, and recalling that each coarse interval has two subintervals of length $h / 2$, we obtain

$$
\begin{aligned}
\tilde{F}(h) & =\sum_{K_{h}} \frac{h}{2}\left(\frac{h}{4}\right)^{2} \frac{a_{h / 2}^{-}\left(a_{h / 2}^{+}\right)^{2}+a_{h / 2}^{+}\left(a_{h / 2}^{-}\right)^{2}}{\left(\frac{a_{h / 2}^{+}+a_{h / 2}^{-}}{2}\right)^{2}} D^{2} u_{h / 2} D^{2} \lambda_{h / 2} \\
& =\sum_{K_{h}} \frac{h^{3}}{16} \frac{2}{\frac{1}{a_{h / 2}^{+}}+\frac{1}{a_{h / 2}^{-}}} D^{2} u_{h / 2} D^{2} \lambda_{h / 2}=: \sum_{K_{h}} \frac{h^{3}}{16} a_{h}^{*} D^{2} u_{h / 2} D^{2} \lambda_{h / 2},
\end{aligned}
$$

where $a_{h}^{*}$ is the harmonic mean of $a_{h / 2}$ over each coarse interval.

Thus, given assumption (4.2), Theorem 4.4 suggests a computable estimate for the observable of the Galerkin error for problem (1.1). In practice, we estimate the observable for the Galerkin error by

$$
\mathcal{E}^{h}(g) \leq C^{\prime} F(h) \leq C E_{\text {est }}^{h}(g),
$$

where the estimator,

$$
E_{\text {est }}^{h}(g):=\sum_{K_{h}} \frac{h^{3}}{16} a_{h}^{*}\left|D^{2} u_{h / 2} D^{2} \lambda_{h / 2}\right|,
$$

is a sum over $h$-elements, $K_{h}, C^{\prime}$ is a constant determined from (4.4), and $C=C(\omega)$ is a positive random variable. Note that the functional $E_{\text {est }}^{h}$ depends implicitly on $g$ through the dual variable $\lambda$, and here, and in the sequel, it will be useful to include the superscript $h$ to distinguish between the pathwise Galerkin errors of different mesh sizes. With these ideas in mind, we investigate in $\S 4.3$ the $C$ appearing in (4.17) and determine that a constant factor is suitable for producing a reliable estimator.

Remark 2. The estimator $E_{\text {est }}^{h}(g)$, based on $F(h)$, relies on the computed solutions $u_{h / 2}$ and $\lambda_{h / 2}$. From an applications perspective, it is not unreasonable to use $E_{\text {est }}^{2 h}$ instead of $E_{\text {est }}^{h}$ as an upper-bound predictor for $\mathcal{E}^{h}$. We note that, for the simple model problem, the ratio $\tilde{F}(2 h) / \tilde{F}(h) \approx 2$ since both $D^{2} u_{h}$ and $D^{2} \lambda_{h}$ are $O_{P}\left(h^{-\frac{1}{2}}\right)$.

4.3. Numerical tests for the simple model problem. In the previous section, we derived a computable estimator for the Galerkin error observable given by (4.17) where $C=C(\omega)$ is a random positive constant. Next, we determine a choice of $C$ that produces a reliable estimator by sampling the ratio of $\mathcal{E}^{h}(g)$ to $E_{\text {est }}^{h}(g)$ for different observables for the Galerkin error for the simple model problem. In numerical experiments, we observe that the sample mean of these realizations provides a reasonable factor in (4.17). We consider the observables $\mathcal{E}^{h}(1)$ and $\mathcal{E}^{h}\left(\delta_{\frac{1}{2}}\right)$ that correspond to computing the integral $\mathcal{E}^{h}(1)=\int_{0}^{1}\left(u-u_{h}\right) \mathrm{d} x$ and the difference of point values $\mathcal{E}^{h}\left(\delta_{\frac{1}{2}}\right)=u(0.5)-u_{h}(0.5)$, Respectively.

We compute the desired samples of $\mathcal{E}^{h}$, using a reference solution on a fine mesh, $h=2^{-17}$, and the associated estimator, $E_{\text {est }}^{h}$, for $M=10^{4}$ sample paths. Here we take $M$ large to ensure that the statistical error is small and emphasize that, in practice, only $O(1)$ samples are needed to have a good hold on the statistical error in our error estimates. Each realization of the conductivity, $a$, is generated on an extremely fine mesh and then a quadrature mesh size $k=2^{-22}$ is used to ensure that the quadrature error is negligible. Figure 5 shows the sample distribution of $C$, where the areas of the histogram bins have been normalized to 1 , corresponding to 

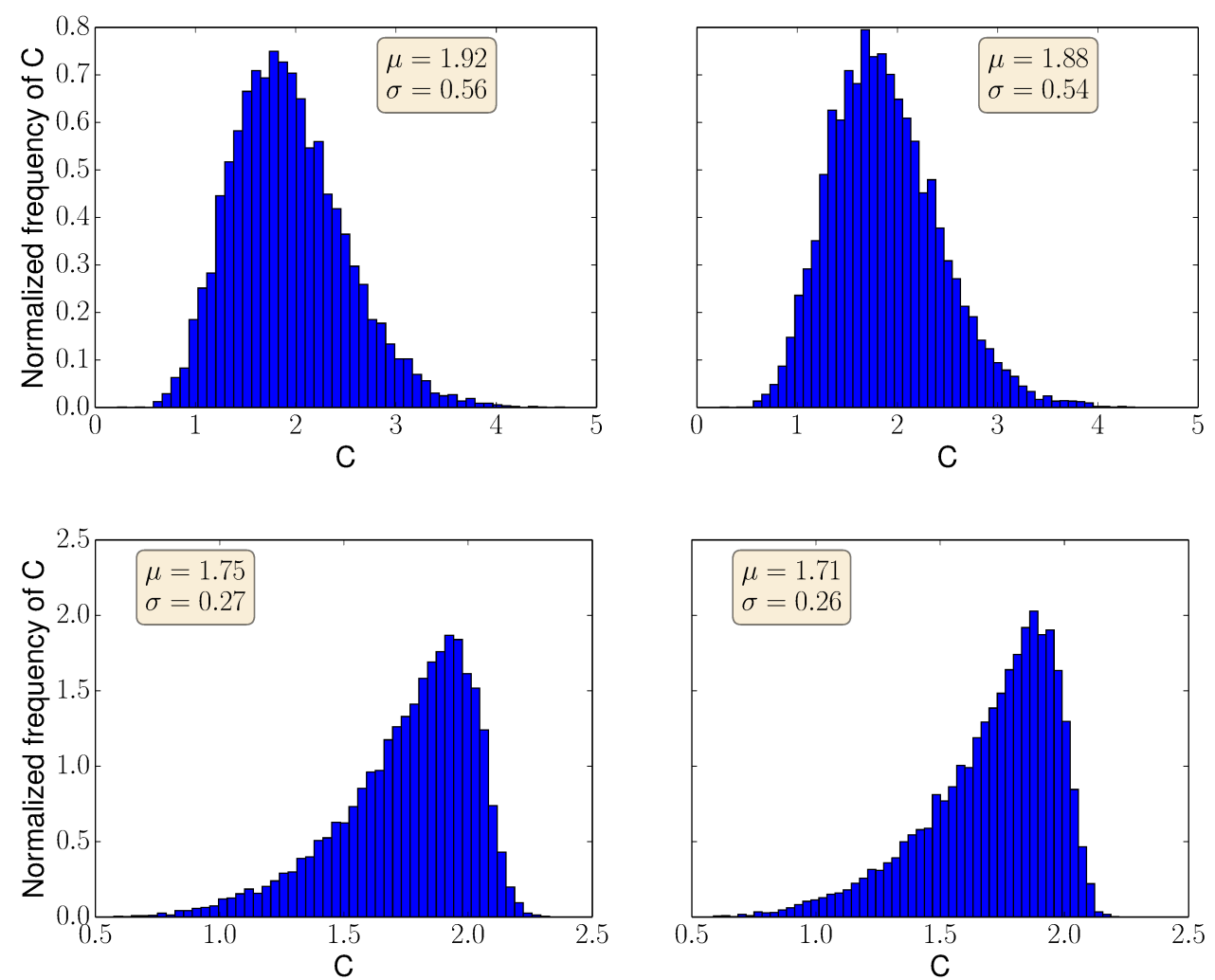

Figure 5: Histogram sample distribution of $C=\left|\mathcal{E}^{h}(g)\right| / E_{\text {est }}^{h}(g)$ for different combinations of mesh sizes and observables. All histograms are computed from $10^{4}$ realizations of $C$ and the areas of the histogram bins are normalized to 1 . The sample distributions for $\mathcal{E}^{h}(1)$ (top row) and $\mathcal{E}^{h}\left(\delta_{\frac{1}{2}}\right)$ (bottom row) are based on finite element solutions of $u_{h}$ on mesh sizes $h=2^{-10}$ (left-hand column) and $h=2^{-12}$ (right-hand column). The results suggests a reliable estimator for the simple model problem (1.1) can be attained using a constant $C=2$.

the observable $g=1$ (top row) and the observable $g=\delta_{\frac{1}{2}}$ (bottom row) computing using the values $h=2^{-10}$ (left column) and $h=2^{-12}$ (right column). The sample mean, $\mu$, and the sample standard deviation, $\sigma$, are also displayed. We observe, for both observables, the sample mean is approximately 2 .

In Figure 6 , we test the fit of using $C=2$, independently of $\omega$, with the estimators for two different observables, $g=1$ (top row) and $g=\delta_{\frac{1}{2}}$ (bottom row). This comparison is done in mean, based on 250 samples, in the left-hand column and is done pathwise in the right-hand column, corresponding to an error and estimator pair drawn uniformly at random from the previously generated realizations. Choosing $C=$ 2 in (4.17) reliably estimates the observable Galerkin error for the generic observables $g=1$ and $g=\delta_{\frac{1}{2}}$. Once again, to explore the limits of the estimator, we consider the observable $g=\cos (2 \pi \cdot)$, a non-generic choice selected to exhibit cancellations between the local indicators. In Figure 7, we plot the error and estimator, using $C=2$, in expectation, based on 250 samples, (left-hand panel) and pathwise (right-hand panel). 

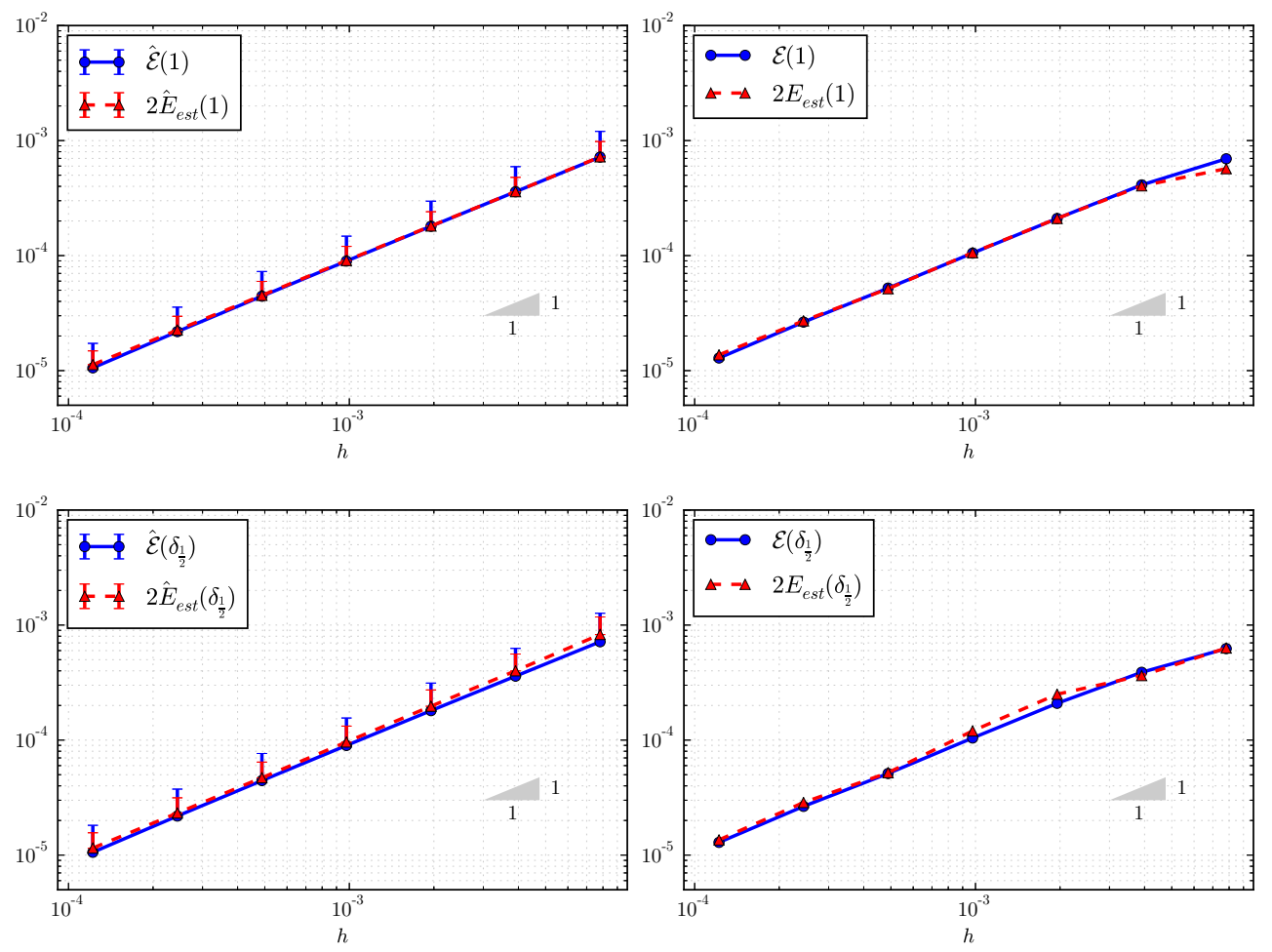

Figure 6: Using a constant $C=2$, independent of $\omega$, produces a reliable estimator for the Galerkin error for the simple model problem (1.1) for the generic observables $g=1$ (top row) and $g=\delta_{\frac{1}{2}}$ (bottom row) both in expectation, based on 250 samples with error bars indicating two standard deviations, (left-hand column) and pathwise for one realization (right-hand column).

As anticipated, the estimator, which is the sum of local error indicators, can become small arbitrarily due to cancellations. In the next section, $\S 5$, we introduce and test an estimator for a two-dimensional model problem, but first we provide heuristics to motivate our use of the pathwise Galerkin error.

4.4. The Pathwise Galerkin error rate is arbitrarily close to the expected Galerkin error rate. In the preceding we studied the pathwise Galerkin error rather than the expected Galerkin error. Due to possible stochastic cancellations, one might suspect that the convergence rate of the expected Galerkin error to be higher than that of the pathwise Galerkin error. We now study the relationship between these rates in detail for the following random PDE that is related to the model problem (1.1), but slightly easier to analyze in this context:

$$
-\left(a(\omega, x) u^{\prime}(\omega, x)\right)^{\prime}=0,
$$

$P$-a.s. for $(\omega, x) \in \Omega \times[0,1]$ subject to the boundary conditions $u(\omega, 0)=0$ and $a(\omega, 1) u^{\prime}(\omega, 1)=1, P$-a.s., with random field $a=e^{W}$, where $W$ denotes a standard Wiener process. The next theorem shows that under certain assumptions on the 

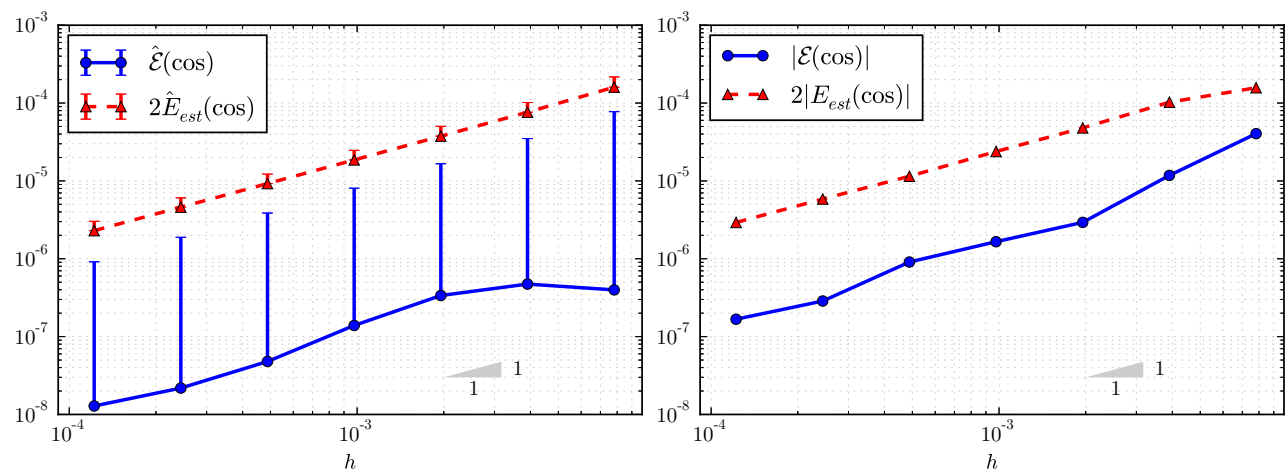

Figure 7: The expected estimator (left-hand panel) and the pathwise estimator for one realization (right-hand panel) for the Galerkin error for the simple model problem for the pathological observable, $g=\cos (2 \pi \cdot)$, can become small due to cancellations. Here the expected estimator is based on 250 samples with error bars indicating two standard deviations.

observable applied to problem (4.18), the pathwise Galerkin error rate is arbitrarily close to the expected Galerkin error rate.

TheOREm 4.5. Consider the random PDE (4.18) and let $u_{h}$ denote the discrete solution on the Galerkin space defined in Section 4.2. For a given observable g, assume that the function $G$, as defined in $(4.9)$, is in $C^{1}([0,1])$, non-negative, and strictly positive on a positive measure subset of $[0,1]$. Then, for any $\delta>0$, there exists a pathwise constant $c \in[0, \infty)$ such that

$$
\left|\left(u-u_{h}, g\right)\right| \leq c h^{1-\delta},
$$

and another constant $c^{\prime}>0$ such that

$$
\lim _{h \rightarrow 0} h^{-1} \mathbf{E}\left[\left(u-u_{h}, g\right)\right]=\int_{0}^{1} \frac{e^{x / 2} G(x)}{6} \mathrm{~d} x>c^{\prime} .
$$

Proof. One may verify that the derivatives of the discrete primal and dual solutions for the considered problem satisfy equation (4.10) in Lemma 4.3. Introducing the mesh points $x_{n}=n h$, for $n=0,1, \ldots h^{-1}=: N$, the pathwise Galerkin error, $\mathcal{E}(g)=\left(u-u_{h}, g\right)$, takes the form

$$
\begin{aligned}
\mathcal{E}(g)= & \int_{0}^{1} a\left(u-u_{h}\right)^{\prime}\left(\lambda-\lambda_{h}\right)^{\prime} \mathrm{d} x \\
= & \int_{0}^{1} a\left(a^{-1}-a_{h}^{-1}\right)^{2} G_{h}(x) \mathrm{d} x+\int_{0}^{1}\left(\frac{1}{a}-\frac{1}{a_{h}}\right)\left(G-G_{h}\right) \mathrm{d} x \\
= & \sum_{n=0}^{N-1} \int_{x_{n}}^{x_{n+1}} a(x)\left(a^{-1}(x)-a\left(\xi_{n}\right)^{-1}\right)^{2} G_{h}(x) \mathrm{d} x \\
& \quad+\sum_{n=0}^{N-1} \int_{x_{n}}^{x_{n+1}}\left(\frac{1}{a}-\frac{1}{a_{h}}\right)\left(G-G_{h}\right) \mathrm{d} x \\
= & I+I I,
\end{aligned}
$$


where $G_{h}(x):=\int_{x_{n}}^{x_{n+1}} G(y) \mathrm{d} y$ and $a_{h}(x)=h^{-1} \int_{x_{n}}^{x_{n+1}} a(y) \mathrm{d} y=a\left(\xi_{n}\right)$ when $x_{n} \leq$ $x<x_{n+1}$, and for some path dependent $\xi_{n} \in\left[x_{n}, x_{n+1}\right]$. By $a \in C^{\frac{1}{2}-\delta}([0,1])$ and $G \in C^{1}([0,1])$, the error terms in (4.20) satisfy $|I| \leq c h^{1-2 \delta}$ and $|I I| \leq c h^{3 / 2-\delta}$, so the Galerkin error has the upper bound

$$
|\mathcal{E}(g)| \leq c h^{1-2 \delta},
$$

for a pathwise dependent constant $c \in[0, \infty)$ and $h$ sufficiently small.

For verifying inequality (4.19), we split, as above, the expected Galerkin error as

$$
\mathbf{E}[\mathcal{E}(g)]=\mathbf{E}\left[\int_{0}^{1}\left(\frac{1}{a}-\frac{1}{a_{h}}\right)\left(G-G_{h}\right) \mathrm{d} x\right]+\mathbf{E}\left[\int_{0}^{1} a\left(a^{-1}-a_{h}^{-1}\right)^{2} G_{h} \mathrm{~d} x\right] .
$$

Using the representation $a^{-1}\left(x_{n}+y\right)=e^{-W\left(x_{n}+y\right)}=e^{-W\left(x_{n}\right)-\left(W\left(x_{n}+y\right)-W\left(x_{n}\right)\right)}$, for $y \in[0, h]$ and $n=0,1, \ldots, N-1$, we proceed computing the second part of the expected Galerkin error (4.21):

$$
\begin{aligned}
\mathbf{E}\left[\int_{0}^{1} a\left(a^{-1}-a_{h}^{-1}\right)^{2} G_{h} \mathrm{~d} x\right] \\
=\sum_{n=0}^{N-1} \mathbf{E}\left[\int_{0}^{h} a\left(x_{n}+y\right)\left(a^{-1}\left(x_{n}+y\right)-a_{h}^{-1}\right)^{2} G_{h}\left(x_{n}+y\right) \mathrm{d} y\right] \\
=\sum_{n=0}^{N-1} \mathbf{E}\left[e^{-W\left(x_{n}\right)} G_{h}\left(x_{n}\right) \int_{0}^{h} e^{W\left(x_{n}+y\right)-W\left(x_{n}\right)}\right. \\
\left.\quad \times\left(e^{-\left(W\left(x_{n}+y\right)-W\left(x_{n}\right)\right)}-\frac{1}{h^{-1} \int_{0}^{h} e^{W\left(x_{n}+z\right)-W\left(x_{n}\right)} \mathrm{d} z}\right)^{2} \mathrm{~d} y\right] \\
=\sum_{n=0}^{N-1} \mathbf{E}\left[e^{-W\left(x_{n}\right)} G_{h}\left(x_{n}\right)\right] \mathbf{E}\left[\int_{0}^{h} a(y)\left(a^{-1}(y)-a_{h}^{-1}\right)^{2} \mathrm{~d} y\right],
\end{aligned}
$$

where we used in the third equality that $W\left(x_{n}\right)$ and $W\left(x_{n}+y\right)-W\left(x_{n}\right)$ are independent Wiener increments. Similarly we have that the first part of the expected Galerkin error (4.21) can be bounded as

$$
\begin{gathered}
\left|\mathbf{E}\left[\int_{0}^{1}\left(\frac{1}{a}-\frac{1}{a_{h}}\right)\left(G-G_{h}\right) \mathrm{d} x\right]\right| \leq \max _{0 \leq x \leq 1}\left|G-G_{h}\right| \mathbf{E}\left[\int_{0}^{1}\left(\frac{1}{a}-\frac{1}{a_{h}}\right) \mathrm{d} x\right] \\
=\max _{0 \leq x \leq 1}\left|G-G_{h}\right| \sum_{n=0}^{N-1} \mathbf{E}\left[e^{-W\left(x_{n}\right)}\right] \mathbf{E}\left[\int_{0}^{h}\left(\frac{1}{a}-\frac{1}{a_{h}}\right) \mathrm{d} y\right] .
\end{gathered}
$$

Furthermore,

$$
\int_{0}^{h} a\left(\frac{1}{a}-\frac{1}{a_{h}}\right)^{2} \mathrm{~d} x=\int_{0}^{h}\left(\frac{1}{a}-\frac{2}{a_{h}}+\frac{a}{a_{h}^{2}}\right) \mathrm{d} x=\int_{0}^{h} \frac{1}{a}-\frac{1}{a_{h}} \mathrm{~d} x .
$$

We now show that the expected value of the integral in (4.24) is given by $\frac{h^{2}}{6}+O\left(h^{5 / 2}\right)$. The expected value of the first term in the last integral in (4.24) is given by

$$
\mathbf{E}\left[\int_{0}^{h} \frac{1}{a} \mathrm{~d} x\right]=\int_{0}^{h} \mathbf{E}\left[e^{-W(x)}\right] \mathrm{d} x=\int_{0}^{h} e^{x / 2} \mathrm{~d} x=2\left(e^{h / 2}-1\right) .
$$


The expected value of the second term in the last integral in (4.24) is given by

$$
\begin{gathered}
\mathbf{E}\left[\int_{0}^{h} \frac{1}{a_{h}} \mathrm{~d} x\right]=h \mathbf{E}\left[\frac{1}{a_{h}}\right]=h \mathbf{E}\left[\left(\frac{1}{h} \int_{0}^{h} e^{W(x)} \mathrm{d} x\right)^{-1}\right] \\
=h \mathbf{E}\left[(\underbrace{\frac{1}{h} \int_{0}^{h}\left(e^{W(x)}-1\right) \mathrm{d} x}_{=: z}+1)^{-1}\right]=h \mathbf{E}\left[1-z+z^{2}-\frac{z^{3}}{1+z}\right] .
\end{gathered}
$$

As before, $\mathbf{E}[z]=\frac{2}{h}\left(e^{h / 2}-1\right)-1$. The next term is given by

$$
\begin{aligned}
\mathbf{E}\left[z^{2}\right] & =\frac{1}{h^{2}} \mathbf{E}\left[\left(\int_{0}^{h}\left(e^{W(x)}-1\right) \mathrm{d} x\right)^{2}\right] \\
& =\frac{1}{h^{2}} \mathbf{E}\left[\int_{0}^{h} \int_{0}^{h}\left(e^{W(x)+W(y)}-e^{W(x)}-e^{W(y)}-1\right) \mathrm{d} x \mathrm{~d} y\right] .
\end{aligned}
$$

Using that $\mathbf{E}\left[(W(x)+W(y))^{2}\right]=x+y+2 \min (x, y)$ and Taylor expansion give

$$
\begin{aligned}
\mathbf{E}\left[z^{2}\right] & =\frac{1}{h^{2}} \int_{0}^{h} \int_{0}^{h}\left(\frac{x+y}{2}+\min (x, y)-\frac{x}{2}-\frac{y}{2}\right) \mathrm{d} x \mathrm{~d} y+O\left(h^{2}\right) \\
& =\frac{1}{h^{2}} \int_{0}^{h} \int_{0}^{h} \min (x, y) \mathrm{d} x \mathrm{~d} y+O\left(h^{2}\right)=\frac{h}{3}+O\left(h^{2}\right) .
\end{aligned}
$$

The last term in (4.25) can be bounded using Cauchy-Schwarz:

$$
\left|\mathbf{E}\left[\frac{z^{3}}{1+z}\right]\right| \leq \sqrt{\mathbf{E}\left[z^{6}\right]} \sqrt{\mathbf{E}\left[\frac{1}{(1+z)^{2}}\right]}=\sqrt{\mathbf{E}\left[z^{6}\right]} \sqrt{\mathbf{E}\left[\frac{1}{a_{h}^{2}}\right]} .
$$

Both factors can be treated using Jensen's inequality:

$$
\mathbf{E}\left[z^{6}\right]=\mathbf{E}\left[\left(\frac{1}{h} \int_{0}^{h}\left(e^{W(x)}-1\right) \mathrm{d} x\right)^{6}\right] \leq \frac{1}{h} \int_{0}^{h} \mathbf{E}\left[\left(e^{W(x)}-1\right)^{6}\right] \mathrm{d} x=O\left(h^{3}\right)
$$

and

$$
\mathbf{E}\left[\frac{1}{a_{h}^{2}}\right]=\mathbf{E}\left[\left(\frac{1}{h} \int_{0}^{h} e^{W(x)} \mathrm{d} x\right)^{-2}\right] \leq \mathbf{E}\left[\frac{1}{h} \int_{0}^{h} e^{-2 W(x)} \mathrm{d} x\right]=\frac{e^{h}-1}{h}=O(1) .
$$

Hence

$$
\begin{aligned}
\mathbf{E}\left[\int_{0}^{h}\left(\frac{1}{a}-\frac{1}{a_{h}}\right) \mathrm{d} x\right] & =2\left(e^{h / 2}-1\right)-h\left(1-\left(\frac{2}{h}\left(e^{h / 2}-1\right)-1\right)+\frac{h}{3}\right)+O\left(h^{5 / 2}\right) \\
& =\frac{h^{2}}{6}+O\left(h^{5 / 2}\right) .
\end{aligned}
$$



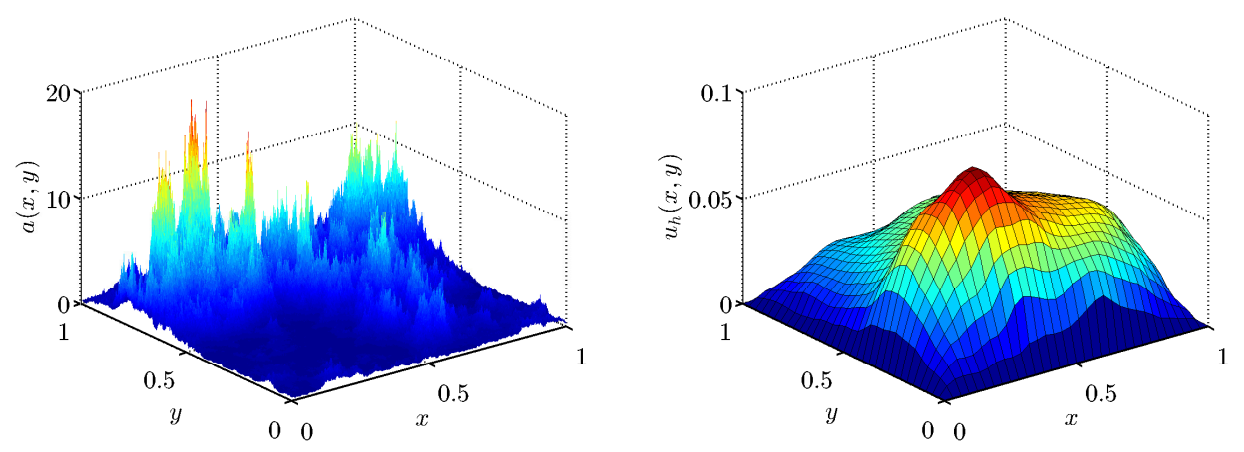

Figure 8: One sample of a rough lognormal conductivity, $a(x, y)$, with covariance (5.2) with $\ell=0.2$ and $\sigma^{2}=1$, generated using a circulant embedding method (lefthand panel) and the corresponding pathwise finite element approximation to problem (5.1) (right-hand panel). For conductivities arising in groundwater flow problems the typically short correlation lengths involved motivate the use of $\mathrm{MC}$ finite element methods over stochastic Galerkin methods.

By equations (4.22), (4.23), (4.26), the equality $\mathbf{E}\left[e^{W(x)}\right]=e^{x / 2}$, and that $G \in$ $C^{1}([0,1])$, we conclude that

$$
\lim _{h \rightarrow 0} h^{-1} \mathbf{E}\left[\left(u-u_{h}, g\right)\right]=\frac{1}{6} \int_{0}^{1} e^{x / 2} G(x) \mathrm{d} x>c^{\prime},
$$

where the inequality follows from $G$ being non-negative and strictly positive on a positive measure subset of $[0,1]$.

5. Two-dimensional numerical experiments. We consider as a model the problem of finding $u \in \mathcal{H}^{1}(\Gamma)$ for $\Gamma=(0,1)^{2}$ such that

$$
-\operatorname{div}(a(x) \nabla u(x))=1 \quad \text { for } x \in \Gamma
$$

subject to the homogeneous boundary condition $u(x)=0$ for all $x \in \partial \Gamma$. We assume the conductivity $a$ is given and that $\log a$ has an exponential covariance given by the two-point covariance function

$$
\operatorname{Cov}(x, y)=\sigma^{2} e^{-\|x-y\| / \ell}
$$

for a given variance, $\sigma^{2}$, and correlation length, $\ell$. For our numerical experiments, we use the circulant embedding method (see for example [2]) to generate samples of a lognormal field $a$ having the desired covariance structure. In Figure 8, one realization of $a(x, y)$, with parameters $\ell=0.2$ and $\sigma^{2}=1$, on a uniform mesh of size $k=2^{-10}$ is plotted (left-hand panel) together with the corresponding finite element approximation, $u_{h}(x, y)$, of problem (5.1) with mesh size $h=2^{-5}$ (right-hand panel). The approximation uses piecewise linear elements, $K \in \mathcal{T}^{h}$, the standard three-point triangulation of $\Gamma$ mesh with size $h$.

The considerations in $\S 4$ suggest that, in practice,

$$
\mathcal{E}^{h}(g) \leq C \int_{\Gamma} a_{h}\left|\nabla\left(u_{h / 2}-u_{h}\right) \cdot \nabla\left(\lambda_{h / 2}-\lambda_{h}\right)\right| \mathrm{d} x
$$


admits an estimator based on local error indicators $a_{h}, \nabla u_{h / 2}, \nabla u_{h}, \nabla \lambda_{h / 2}$, and $\nabla \lambda_{h}$. We approximate this error by the estimator

$$
E_{\text {est }}^{h}(g):=\frac{1}{2} h^{2} \sum_{K} a_{h} \sum_{i=1,2}\left|\partial_{x_{i}}\left(u_{h / 2}-u_{h}\right) \partial_{x_{i}}\left(\lambda_{h / 2}-\lambda_{h}\right)\right|
$$

where $K \in \mathcal{T}^{h}$ and $\partial_{x_{i}}$ is the derivative in the $x_{i}$ direction. In addition, we compare with a second estimator

$$
E_{r e g}^{h}(g):=\frac{1}{2} h^{2} \sum_{K} \frac{h^{2}}{16} a_{h} \sum_{i=1,2}\left|D_{i}^{2} u_{h} \cdot D_{i}^{2} \lambda_{h}\right| .
$$

The quantity $E_{r e g}^{h}(g)$ is proven in [37] to be an asymptotically exact estimate of the Galerkin approximation error, as the mesh size tends to zero, in the case the solution is sufficiently regular, i.e. if $a \in \mathcal{C}^{1}(\bar{\Gamma}), u \in \mathcal{C}^{3}(\bar{\Gamma})$ and $\lambda \in \mathcal{C}^{3}(\bar{\Gamma})$, using bilinear finite elements with possible hanging node refinements. The asymptotic exactness of $E_{\text {reg }}^{h}$, which is related $E_{e s t}^{h}$ by constant factor of order one, shows, for instance, that the error estimator does not miss the contribution from the jumps of the derivatives at the edges, although these are not explicitly present in the estimator.

In Figure 9, we plot $\mathcal{E}^{h}(1)$, using a reference mesh of $h=2^{-10}$, and the estimators $E_{\text {est }}^{h}(1)$ and $E_{\text {reg }}^{h}(1)$, all using a quadrature mesh, $k=2^{-13}$. This figure demonstrates that the estimator $E_{\text {est }}^{h}$, based on local error indicators, gives an estimate of the error that is realized pathwise provided that one has the quadrature error under control. We choose $C=1$ to show that the estimator holds with a nonrandom constant, keeping in mind that this choice of constant is ad hoc and does not represent a best fit. Further, we note that the Galerkin error for the problem with rough lognormal conductivity is on the order of $h$. On the other hand Figure 9 also shows, as expected, that $E_{\text {reg }}^{h}$ misses the high frequency error with a factor in the case that the solutions have low regularity, analogously to the high- and low-frequency estimators in Figure 1. Estimates for the observable quadrature error are the subject of the next section.

6. Quadrature error. The numerical experiments that we have presented thus far focused solely on the Galerkin error at the expense of overkilling the quadrature error. Next, we consider the problem of estimating the expected quadrature error when computing an observable using a piecewise linear finite element approximation of problem (2.1). If the conductivity $a$ enjoys a certain degree of regularity, then a quadrature rule can be chosen such that its error contribution is negligible compared with the Galerkin error, as is typically the case in the deterministic setting. In contrast, when a conductivity $a$ has a rough lognormal distribution such as the log-Brownian bridge distribution used in (1.1), Figure 10 suggests that the quadrature error is on the same order as the Galerkin error (cf. Figure 1, where the rate of the Galerkin error is plotted). In the present setting, it is therefore pertinent to construct an estimator for the expected quadrature error committed in calculating an observable.

To discuss the quadrature error, we first present some additional notation. Recall that a finite element solution, $u_{h} \in V_{h}$, to (2.1) satisfies

$$
\int_{\Gamma} a_{h}(x) \nabla u_{h}(x) \cdot \nabla v_{h}(x) \mathrm{d} x=\int_{\Gamma} f(x) v_{h}(x) \mathrm{d} x \quad P \text {-a.s. }
$$

for all $v_{h} \in V_{h} \subset V$, where $a_{h}$ is the spatial average of $a$ over each $h$-element $K_{h}$, as defined in (4.5). Here, we assume that the finite element solution, $u_{h}$, is computed 


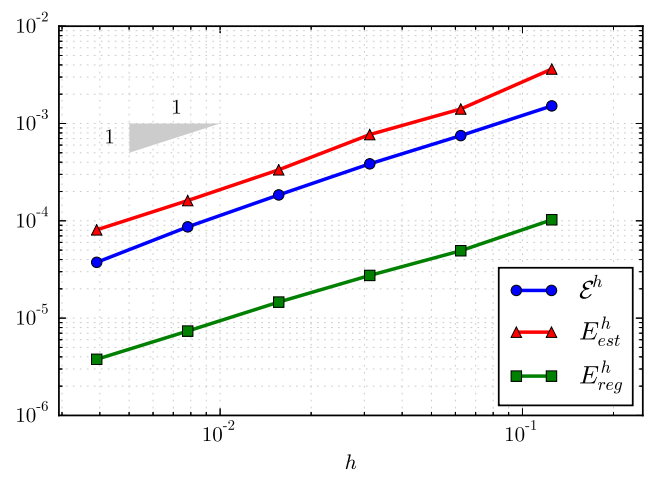

Figure 9: For the two-dimensional test problem (5.1), the estimator, $E_{\text {est }}^{h}(1)$, constructed from local error indicators reliably estimates the pathwise Galerkin error, $\mathcal{E}^{h}(1)$, with a constant factor independently of $\omega$. As expected, $E_{r e g}^{h}(1)$ misses the high frequency error with a factor, but the asymptotic exactness demonstrates that the estimator does not miss the contribution form the jumps of the derivatives at the edges of elements although they are not explicitly present.

without quadrature error. That is, we assume that the components of the stiffness matrix, which are of the form

$$
\int_{\Gamma} a_{h}(x) \nabla \phi_{i}(x) \cdot \nabla \phi_{j}(x) \mathrm{d} x
$$

are computed exactly. Likewise, an exact variational formulation of the discrete dual, $\lambda_{h} \in V_{h}$, satisfies

$$
\int_{\Gamma} a_{h}(x) \nabla \lambda_{h}(x) \cdot \nabla v_{h}(x) \mathrm{d} x=\int_{\Gamma} g(x) v_{h}(x) \mathrm{d} x \quad P \text {-a.s. }
$$

for all $v_{h} \in V_{h} \subset V$.

Together with (6.1) and (6.2), we consider formulations where a quadrature rule is used in the assembly of the stiffness matrix in the finite element problem. We subdivide the $h$-mesh, of elements $K_{h}$, into a quadrature $k$-mesh, of elements $K_{k} \subset$ $K_{h}$, with mesh size $k=k(h, n)=h 2^{-n}$, for some $n \in \mathbf{N}:=\{0,1,2, \ldots\}$. On the $k$-mesh, we define the functions $\bar{a}_{h, k}, \bar{f}_{h, k}: \Gamma \rightarrow \mathbf{R}$ which are piecewise constant on all $K_{k}$ elements

$$
\bar{a}_{h, k}(x):=\sum_{K_{k}} a\left(x_{K_{k}}\right) \mathbf{1}_{K_{k}}(x) \quad \text { and } \quad \bar{f}_{h, k}(x):=\sum_{K_{k}} f\left(x_{K_{k}}\right) \mathbf{1}_{K_{k}}(x),
$$

where $x_{K_{k}}$ is the midpoint (or a corner point) of the element $K_{k}$. Taking averages over the $h$-elements, we have the piecewise constant functions $a_{h, k}$ and $f_{h, k}: \Gamma \rightarrow \mathbf{R}$ defined by

$$
a_{h, k}(x):=\sum_{K_{k} \subset K_{h}} a\left(x_{K_{k}}\right)\left|K_{k}\right| /\left|K_{h}\right| \quad \text { and } \quad f_{h, k}(x):=\sum_{K_{k} \subset K_{h}} f\left(x_{K_{k}}\right)\left|K_{k}\right| /\left|K_{h}\right|,
$$

for $x \in K_{h}$, where $|K|=\int_{K} \mathrm{~d} x$ is the size of the element $K$. Then in variational form, we seek $u_{h, k} \in V_{h}$ such that

$$
\int_{\Gamma} a_{h, k}(x) \nabla u_{h, k}(x) \cdot \nabla v_{h}(x) \mathrm{d} x=\int_{\Gamma} f_{h, k}(x) v_{h}(x) \mathrm{d} x \quad P \text {-a.s. }
$$


for all $v_{h} \in V_{h}$ and, similarly, we seek $\lambda_{h, k} \in V_{h}$ such that

$$
\int_{\Gamma} a_{h, k}(x) \nabla \lambda_{h, k}(x) \cdot \nabla v_{h}(x) \mathrm{d} x=\int_{\Gamma} g v_{h} \mathrm{~d} x \quad P \text {-a.s. }
$$

for all $v_{h} \in V_{h} \subset V$, which corresponds to using the quadrature rule for calculating the components of the stiffness matrix. As the regularity of $a$ is low, the midpoint rule with a fixed number of points per $h$-element is a reasonable strategy for approximating the spatial average of $a$.

We are interested in estimating

$$
\mathbf{E} \mathcal{Q}(g):=\mathbf{E}\left[\left(g, u_{h}-u_{h, k}\right)\right],
$$

the expected quadrature error in the finite element approximation of (2.1). For a finite sample of size $M$, we will consider the MC approximation

$$
\hat{\mathcal{Q}}^{h, k}(g)=\frac{1}{M} \sum_{m=1}^{M}\left(g, u_{h}\left(\omega_{m}\right)-u_{h, k}\left(\omega_{m}\right)\right)
$$

The statistical error committed in the MC approximation is $O_{P}\left(\sigma_{s} / \sqrt{M}\right)$ where $\sigma_{s}$ is the standard deviation of $\left(g, u_{h}-u_{h, k}\right)$. This extra statistical error contribution can be made small, relative to the contributions from the Galerkin error and the quadrature error, by choosing sufficiently large $M$. In the next theorem we demonstrate that once again an assumption on scales and a telescoping argument can be used to obtain an estimator for the desired expected quadrature error.

Theorem 6.1. Assume that for positive constants $C$ and $\gamma$,

$$
\left|\mathbf{E}\left[\int_{\Gamma}\left(a_{h, k}-a_{h, k / 2}\right) \nabla u_{h, k} \cdot \nabla \lambda_{h} \mathrm{~d} x\right]\right|<C k^{\gamma},
$$

that for all sufficiently small $h, k>0$,

$$
\lim _{j \rightarrow \infty} \mathbf{E}\left[\int_{\Gamma}\left(a_{h, k 2^{-j}}-a_{h}\right) \nabla u_{h, k} \cdot \nabla \lambda_{h} \mathrm{~d} x\right]=0,
$$

and that

$$
\lim _{k \rightarrow 0} k^{-\gamma} \mathbf{E}\left[\int_{\Gamma}\left(f-f_{h, k}\right) \lambda_{h} \mathrm{~d} x\right]=0
$$

Then there is a constant $\hat{C}$ such that

$$
\left|\mathbf{E}\left[\int_{\Gamma} g\left(u_{h}-u_{h, k}\right) \mathrm{d} x\right]\right| \leq \frac{\hat{C} k^{\gamma}}{1-2^{-\gamma}}
$$

Proof. Using (6.2), (6.1), and (6.3), the expected quadrature error can be ex- 
pressed as

$$
\begin{aligned}
\mathbf{E}\left[\int_{\Gamma} g\left(u_{h}-u_{h, k}\right) \mathrm{d} x\right] & =\mathbf{E}\left[\int_{\Gamma}\left(a_{h} \nabla \lambda_{h}\right) \cdot \nabla\left(u_{h}-u_{h, k}\right) \mathrm{d} x\right] \\
& =\mathbf{E}\left[\int_{\Gamma} a_{h} \nabla u_{h} \cdot \nabla \lambda_{h} \mathrm{~d} x-\int_{\Gamma} a_{h} \nabla u_{h, k} \cdot \nabla \lambda_{h} \mathrm{~d} x\right] \\
& =\mathbf{E}\left[\int_{\Gamma} f \lambda_{h} \mathrm{~d} x-\int_{\Gamma} a_{h} \nabla u_{h, k} \cdot \nabla \lambda_{h} \mathrm{~d} x\right] \\
& =\mathbf{E}\left[\int_{\Gamma} f_{h, k} \lambda_{h} \mathrm{~d} x-\int_{\Gamma} a_{h} \nabla u_{h, k} \cdot \nabla \lambda_{h} \mathrm{~d} x\right]+r \\
& =\mathbf{E}\left[\int_{\Gamma}\left(a_{h, k}-a_{h}\right) \nabla u_{h, k} \cdot \nabla \lambda_{h} \mathrm{~d} x\right]+r,
\end{aligned}
$$

where $r=\mathbf{E}\left[\int_{\Gamma}\left(f-f_{h, k}\right) \lambda_{h} \mathrm{~d} x\right]$ is a remainder term appearing in equation (6.5). Looking at the final expression, we expand $\left(a_{h, k}-a_{h}\right)$ in a telescoping series in $k$,

$$
\begin{aligned}
\mid \mathbf{E} & {\left[\int_{\Gamma}\left(a_{h, k}-a_{h}\right) \nabla u_{h, k} \cdot \nabla \lambda_{h} \mathrm{~d} x\right] \mid } \\
& \leq \sum_{j=0}^{\infty}\left|\mathbf{E}\left[\int_{\Gamma}\left(a_{h, k 2^{-j}}-a_{h, k 2^{-(j+1)}}\right) \nabla u_{h, k} \cdot \nabla \lambda_{h} \mathrm{~d} x\right]\right| \\
& \leq \sum_{j=0}^{\infty} C k^{\gamma} 2^{-\gamma j}=\frac{C k^{\gamma}}{1-2^{-\gamma}},
\end{aligned}
$$

and the result follows by assumption (6.5) and introducing a constant $\hat{C}>C$ such that

$$
\frac{C k^{\gamma}}{1-2^{-\gamma}}+|r| \leq \frac{\hat{C} k^{\gamma}}{1-2^{-\gamma}}
$$

Theorem 6.1 motivates estimating the observable for the quadrature error by an MC approximation of

$$
\mathbf{E}\left[\int_{\Gamma}\left(a_{h, k}-a_{h, k / 2}\right) \nabla u_{h, k} \cdot \nabla \lambda_{h} \mathrm{~d} x\right] \approx C k^{\gamma}
$$

We remark that, as was the case for Galerkin estimator, here we must also assume that the expected quadrature estimator is bounded from below to formally replace $C k^{\gamma}$ in (6.6) by (6.7) when estimating the observable for the expected quadrature error. However, unlike in the case of the Galerkin error, taking absolute values in the integrand produces an estimator that is too large to be useful in practice. This is due to the presence of cancellations in the observable for the quadrature error even for generic choices of observable $g$.

Putting off the justification for the assumption on scales until Theorem 6.2, we proceed to test the estimator for the one-dimensional model problem (1.1) for the observable $g=1$. For a given sample, $\omega_{m} \in \Omega$, we compute the pathwise estimator

$$
Q_{m}^{h, k}:=\frac{h^{2}}{2} \sum_{K_{h}}\left(a_{h, k}\left(\omega_{m}\right)-a_{h, k / 2}\left(\omega_{m}\right)\right) \nabla u_{h, k}\left(\omega_{m}\right) \cdot \nabla \lambda_{h, \tilde{k}}\left(\omega_{m}\right),
$$


where the sum is over $h$-elements, $K_{h}$, and $\tilde{k}$ is a reference mesh that will receive further attention in the discussion below. In dimension one, we use the trapezoid rule, for convenience, and, in particular, for $k=h$ we have

$$
a_{h, h}(x)=\frac{1}{2}\left(a\left(x_{j}\right)+a\left(x_{j+1}\right)\right),
$$

for $x \in K_{h}=\left[x_{j}, x_{j+1}\right]$, the (spatial) mean value of $a$ on the $h$-element, $K_{h}$. The estimator requires $a_{h, k}$ at two levels of the quadrature mesh: $k=h 2^{-n}$ and $k / 2=$ $h 2^{-(n+1)}$. We then form the MC approximation,

$$
\hat{Q}^{h, k}:=\frac{1}{M} \sum_{m=1}^{M} Q_{m}^{h, k},
$$

for a sample population of size $M$. When necessary, we distinguish between different samples of the MC approximations for the error and the estimator with the addition of a subscript.

Taking a second look at Figure 10, we compare the quadrature error and the estimator for the observable $g=1$ for the one-dimensional model problem (1.1). We compute MC approximations, $\hat{\mathcal{Q}}^{h, h}$ and $\hat{Q}^{h, h}$, for $M=2^{13}$ samples to ensure that the statistical error, which is on the same order as the quantity $\sigma_{M}:=\sigma_{s} / \sqrt{M}$, is negligible compared to the quadrature error (see Table 1). The error observable uses $u_{h}=u_{h, \tilde{k}}$ on a reference quadrature mesh of $\tilde{k}=2^{-24}$ and the estimator is computed using $\lambda_{h}=\lambda_{h, \tilde{k}}$ on a reference quadrature mesh of $\tilde{k}=2^{-22}$. Here, for both the error and the estimator, we take the quadrature mesh to match the finite element mesh so that we can test the sensitivity of the dual solution in the estimator on the reference quadrature mesh. Thus, in Figure 10, we also plot $\hat{Q}_{*}^{h, h}$, a more computationally practical estimator, computed using the discrete dual $\lambda_{h, h / 2}$, with a quadrature mesh of size $\tilde{k}=h / 2$, instead of the reference mesh. We observe that there is no perceivable difference between the estimators $\hat{Q}^{h, h}$ and $\hat{Q}_{*}^{h, h}$. Therefore, we redefine $\hat{Q}^{h, k}:=\hat{Q}_{*}^{h, k}$ and employ the practical estimator, using the discrete dual on the quadrature mesh, $\tilde{k}=k / 2$, in the sequel. Note the constant $C=2$ is not chosen as a best fit but merely to demonstrate that the estimator provides a reliable estimate using a constant factor.

Table 1: The statistical error as measured by $\sigma_{M}:=\sigma_{s} / \sqrt{M}$, based on $M=2^{13}$ samples to ensure that the statistical error is negligible, corresponding to $\hat{\mathcal{Q}}^{h, h}$ (third row) and $\hat{Q}^{h, h}$ (fifth rows) for the observable $g=1$, is small compared to the corresponding expected quadrature error.

\begin{tabular}{|l|rrrrrrrrr|}
\hline $\log _{2} h$ & -13 & -12 & -11 & -10 & -9 & -8 & -7 & -6 & -5 \\
\hline$\hat{\mathcal{Q}}^{h, h}$ & $5.6 \mathrm{e}-6$ & $1.1 \mathrm{e}-5$ & $2.4 \mathrm{e}-5$ & $4.2-5$ & $8.1 \mathrm{e}-5$ & $1.8 \mathrm{e}-4$ & $3.6 \mathrm{e}-4$ & $6.8 \mathrm{e}-4$ & $1.5 \mathrm{e}-3$ \\
$\sigma_{M}$ & $2.6 \mathrm{e}-7$ & $5.3 \mathrm{e}-7$ & $1.1 \mathrm{e}-6$ & $2.1 \mathrm{e}-6$ & $4.2 \mathrm{e}-6$ & $8.6 \mathrm{e}-6$ & $1.7 \mathrm{e}-5$ & $3.4 \mathrm{e}-5$ & $6.7 \mathrm{e}-5$ \\
\hline$\hat{Q}^{h, h}$ & $4.1 \mathrm{e}-6$ & $8.6 \mathrm{e}-6$ & $1.8 \mathrm{e}-5$ & $2.9 \mathrm{e}-5$ & $6.1 \mathrm{e}-5$ & $1.4 \mathrm{e}-4$ & $2.7 \mathrm{e}-4$ & $5.0 \mathrm{e}-4$ & $1.2 \mathrm{e}-3$ \\
$\sigma_{M}$ & $2.3 \mathrm{e}-7$ & $4.6 \mathrm{e}-7$ & $9.3 \mathrm{e}-7$ & $1.8 \mathrm{e}-6$ & $3.4 \mathrm{e}-6$ & $7.4 \mathrm{e}-6$ & $1.5 \mathrm{e}-5$ & $2.9 \mathrm{e}-5$ & $5.8 \mathrm{e}-5$ \\
\hline
\end{tabular}

Next, we proceed to justify the assumption on scales for the quadrature rule. One can use Malliavin calculus arguments to show that (6.4) has the desired decay rate (see the proof of Theorem 3.3 in [39], that provides an estimate for an adaptive timestepping method for SDE, containing a similar argument). In the setting of model 


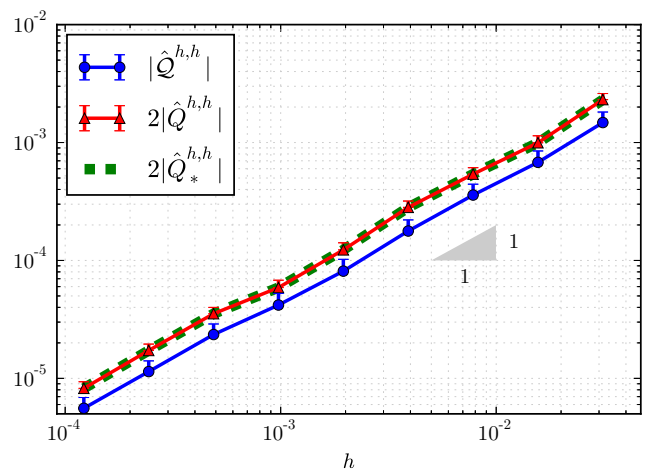

Figure 10: For problem (1.1) for the observable $g=1$, the expected quadrature error committed in the finite element method can be estimated by local error indicators. Here the calculation of the mean is based on $M=2^{13}$ samples to ensure that the statistical error is negligible and the one-sided error bars indicate $5 \sigma_{M}$-deviations from the corresponding mean.

problem (1.1), the argument greatly simplifies if one takes advantage of the available representations of the primal and dual solutions.

THEOREM 6.2. Consider the model problem (1.1) and the corresponding finite element solution $u_{h, k}$ defined by equation (6.3). Assume that the observable $g$ is sufficiently smooth to ensure that $G \in L^{1}(0,1)$, for the primitive function $G$ as defined in (4.9). Then, the quadrature error assumption of scales

$$
\left|\mathbf{E}\left[\int_{\Gamma}\left(a_{h, k}-a_{h, k / 2}\right) \nabla u_{h, k} \cdot \nabla \lambda_{h} \mathrm{~d} x\right]\right|<C k^{\gamma},
$$

holds with $\gamma=1$, provided the forward Euler quadrature rule, (6.8) below, is used to compute the stiffness matrix function $a_{h, k}$.

Proof. One can represent an explicit Euler quadrature rule so that

$$
a_{h, h}(x)=a\left(x_{i}\right)
$$

for $x \in\left[x_{i}, x_{i+1}\right)$, and, more generally,

$$
a_{h, k}(x)=\sum_{j=1}^{h / k} \frac{k}{h} a\left(x_{i, j}\right),
$$

for $x \in\left[x_{i}, x_{i+1}\right)$ and $x_{i, j}:=i h+j k$. As alternatives to $a_{h, k}$, which are piecewise constant functions on intervals of length $h$, it will be sufficient to consider functions $\bar{a}_{h, k}$ that are piecewise constant on intervals of length $k$ such that

$$
\int_{0}^{1}\left(a_{h, k}-\bar{a}_{h, k}\right) \nabla u_{h} \cdot \nabla v_{h} \mathrm{~d} x=0
$$

for all $u, v \in V_{h}$. For explicit Euler quadrature, we obtain that

$$
\bar{a}_{h, k}(x)=e^{\bar{B}_{h, k}(x)}
$$


with

$$
\bar{B}_{h, k}(x)=\sum_{i=1}^{h^{-1}} \sum_{j=1}^{h / k} \mathbf{1}_{\left\{x_{i, j}<x\right\}} \Delta B_{i, j}^{k}
$$

where $\Delta B_{i, j}^{k}$ is an increment of the Brownian bridge over the interval $[i h+(j-1) k, i h+$ $j k)$. Thus, $\bar{a}_{h, k}(x)$ is piecewise constant over $k$-intervals due to the piecewise constant $\bar{B}_{h, k}(x)$. Similarly, we have that

$$
\bar{a}_{h, k / 2}(x)=e^{\bar{B}_{h, k / 2}(x)}
$$

with

$$
\bar{B}_{h, k / 2}(x)=\sum_{i=1}^{h^{-1}} \sum_{j=1}^{2 h / k} \mathbf{1}_{\{i h+j k / 2<x\}} \Delta B_{i, j}^{k / 2} .
$$

For working on the pointwise difference between $\bar{a}_{h, k / 2}-\bar{a}_{h, k}$, we simplify further by reducing to one index, $\ell(i, j)=i 2 h / k+j$, and noting that we may write

$$
\bar{B}_{h, k}(x)=\sum_{\ell \leq 2 k^{-1}} \mathbf{1}_{\{\ell k / 2<x\}} \mathbf{1}_{\{\ell \text { is even }\}}\left(\Delta B_{\ell-1}^{k / 2}+\Delta B_{\ell}^{k / 2}\right)
$$

and

$$
\bar{B}_{h, k / 2}(x)=\sum_{\ell \leq 2 k^{-1}} \mathbf{1}_{\{\ell k / 2<x\}} \Delta B_{\ell}^{k / 2}
$$

Consequently,

$$
\left(\bar{B}_{h, k}-\bar{B}_{h, k / 2}\right)(x)= \begin{cases}-\Delta B_{\lfloor 2 x / k\rfloor}^{k / 2} & \text { if }\lfloor 2 x / k\rfloor \text { is odd. } \\ 0 & \text { else. }\end{cases}
$$

Below we shall test the sensitivity of terms arising in the representation of the quadrature error with respect to the increments of the processes defined above. When convenient, we shall abuse the notation slightly by writing $a\left(\bar{B}_{h, k}(x)\right)=e^{\bar{B}_{h, k}(x)}$ for the log-Brownian bridge path depending on the discrete increments, $\bar{B}_{h, k}(x)$.

Using this newly introduced notation, we apply a Taylor expansion and the Mean Value Theorem to the left-hand side of (6.7) to obtain

$$
\begin{aligned}
\mathbf{E} & {\left[\int_{0}^{1}\left(a\left(\bar{B}_{h, k}(x)\right)-a\left(\bar{B}_{h, k / 2}(x)\right)\right) u_{h, k}^{\prime} \lambda_{h}^{\prime} \mathrm{d} x\right] } \\
= & \mathbf{E}\left[\int_{0}^{1} a^{\prime}\left(\bar{B}_{h, k / 2}\right) u_{h, k}^{\prime} \lambda_{h}^{\prime}\left(\bar{B}_{h, k}-\bar{B}_{h, k / 2}\right) \mathrm{d} x\right] \\
& +\mathbf{E}\left[\frac{1}{2} a^{\prime \prime}\left(\theta \bar{B}_{h, k / 2}+(1-\theta) \bar{B}_{h, k}\right) u_{h, k}^{\prime} \lambda_{h}^{\prime}\left(\bar{B}_{h, k}-\bar{B}_{h, k / 2}\right)^{2} \mathrm{~d} x\right]
\end{aligned}
$$

where $\theta=\theta(x, \omega) \in[0,1]$ denotes a Mean Value Theorem constant. Assuming all needed moments are bounded (a reasonable assumption since, under the slight abuse of notation $a(B)=e^{B}$, we have that $a^{\prime \prime}=a$ in the case of our simple model problem), 
the second summand on the right-hand side of (6.9) may be bounded by Hölder's inequality as follows

$$
\begin{aligned}
\int_{0}^{1} \frac{1}{2} & \mathbf{E}\left[a^{\prime \prime}\left(\theta \bar{B}_{h, k / 2}+(1-\theta) \bar{B}_{h, k}\right)\left(\bar{B}_{h, k}-\bar{B}_{h, k / 2}\right)^{2} u_{h, k}^{\prime} \lambda_{h}^{\prime}\right] \mathrm{d} x \\
\leq & \frac{1}{2} \int_{0}^{1} \sqrt{\mathbf{E}\left[\left(a^{\prime \prime}\left(\theta \bar{B}_{h, k / 2}+(1-\theta) \bar{B}_{h, k}\right) u_{h, k}^{\prime} \lambda_{h}^{\prime}\right)^{2}\right]} \sqrt{\mathbf{E}\left[\left(\bar{B}_{h, k}-\bar{B}_{h, k / 2}\right)^{4}\right]} \mathrm{d} x \\
\leq & C k \int_{0}^{1}\left|G_{h}\right| \mathrm{d} x \leq C k
\end{aligned}
$$

where we have used

$$
\mathbf{E}\left[\left(\bar{B}_{h, k}(x)-\bar{B}_{h, k / 2}(x)\right)^{4}\right]=\mathbf{E}\left[\left(\Delta B_{\lfloor 2 x / k\rfloor}^{k / 2}\right)^{4} \mathbf{1}_{\{\lfloor 2 x / k\rfloor \text { is odd }\}}\right]=O\left(k^{2}\right),
$$

that $\lambda_{h}^{\prime}=G_{h} / a_{h}$, cf. Lemma 4.3 , and that $G_{h} \in L^{1}(0,1)$, which implicitly follows from $G \in L^{1}(0,1)$.

For achieving a bound of the same order for the first summand of (6.9), we begin by noting that

$$
\begin{array}{rl}
\int_{0}^{1} & \mathbf{E}\left[a^{\prime}\left(\bar{B}_{h, k / 2}\right) u_{h, k}^{\prime} \lambda_{h}^{\prime}\left(\bar{B}_{h, k}-\bar{B}_{h, k / 2}\right)\right] d x \\
\quad=\int_{0}^{1} \mathbf{E}\left[a^{\prime}\left(\bar{B}_{h, k / 2}\right) u_{h, k}^{\prime} \mathbf{E}\left[\lambda_{h}^{\prime} \mid \Delta B_{\lfloor 2 x / k\rfloor}^{k / 2}\right] \Delta B_{\lfloor 2 x / k\rfloor}^{k / 2} \mathbf{1}_{\{\lfloor 2 x / k\rfloor \text { is odd }\}}\right] \mathrm{d} x .
\end{array}
$$

We introduce the notation

$$
S\left(\left(\Delta B_{\ell}^{k / 2}\right)_{\ell=1}^{k^{-1}}\right):=a^{\prime}\left(\bar{B}_{h, k / 2}\right) u_{h, k}^{\prime} \mathbf{E}\left[\lambda_{h}^{\prime} \mid \Delta B_{\lfloor 2 x / k\rfloor}^{k / 2}\right]
$$

with the convenient shorthand

$$
\hat{S}(z)=S\left(\Delta B_{1}^{k / 2}, \ldots, \Delta B_{\lfloor 2 x / k\rfloor-1}^{k / 2}, z, \Delta B_{\lfloor 2 x / k\rfloor+1}^{k / 2}, \ldots, \Delta B_{k^{-1}}^{k / 2}\right),
$$

and Taylor expand, with, once again, $\theta=\theta(x, \omega) \in[0,1]$ denoting a Mean Value Theorem coefficient,

$$
\begin{aligned}
S\left(\left(\Delta B_{\ell}^{k / 2}\right)_{\ell=1}^{k^{-1}}\right) & =\hat{S}\left(\Delta B_{\lfloor 2 x / k\rfloor}^{k / 2}\right) \\
& =\hat{S}(0)+\hat{S}^{\prime}\left(\theta \Delta B_{\lfloor 2 x / k\rfloor}^{k / 2}\right) \Delta B_{\lfloor 2 x / k\rfloor}^{k / 2} .
\end{aligned}
$$

Substituting the expansion of $\hat{S}\left(\Delta B_{\lfloor 2 x / k\rfloor}^{k / 2}\right)$ into (6.10), noting that $\hat{S}(0)$ is independent of $\Delta B_{\lfloor 2 x / k\rfloor}^{k / 2}$, and using Hölder's inequality on the other integrand yields

$$
\begin{array}{rl}
\int_{0}^{1} & \mathbf{E}\left[a^{\prime}\left(\bar{B}_{h, k / 2}\right) u_{h, k}^{\prime} \mathbf{E}\left[\lambda_{h}^{\prime} \mid \Delta B_{\lfloor 2 x / k\rfloor}^{k / 2}\right] \Delta B_{\lfloor 2 x / k\rfloor}^{k / 2} \mathbf{1}_{\{\lfloor 2 x / k\rfloor \text { is odd }\}]} \mathrm{d} x\right. \\
= & \int_{0}^{1} \mathbf{E}\left[\left(\hat{S}(0)+\hat{S}^{\prime}\left(\theta \Delta B_{\lfloor 2 x / k\rfloor}^{k / 2}\right) \Delta B_{\lfloor 2 x / k\rfloor}^{k / 2} \Delta B_{\lfloor 2 x / k\rfloor}^{k / 2} \mathbf{1}_{\{\lfloor 2 x / k\rfloor \text { is odd }\}]} \mathrm{d} x\right.\right. \\
\leq & \int_{0}^{1} \mathbf{E}[\hat{S}(0)] \mathbf{E}\left[\Delta B_{\lfloor 2 x / k\rfloor}^{k / 2} \mathbf{1}_{\{\lfloor 2 x / k\rfloor \text { is odd }\}]} \mathrm{d} x\right. \\
& +\int_{0}^{1} \sqrt{\mathbf{E}\left[\left(\hat{S}^{\prime}\left(\theta \Delta B_{\lfloor 2 x / k\rfloor}^{k / 2}\right)\right)^{2}\right]} \sqrt{\mathbf{E}\left[\left(\Delta B_{\lfloor 2 x / k\rfloor}^{k / 2}\right)^{4} \mathbf{1}_{\{\lfloor 2 x / k\rfloor \text { is odd }\}}\right]} \mathrm{d} x \\
\leq & C k
\end{array}
$$


since $\mathbf{E}\left[\Delta B_{\lfloor 2 x / k\rfloor}^{k / 2} \mathbf{1}_{\{\lfloor 2 x / k\rfloor \text { is odd }\}}\right]=0$. Hence the assumption on scales for the quadrature error holds with $\gamma=1$ in the case of the simple model problem (1.1).

7. Discussion and future work. In the present work, we have presented reliable and computable estimates for the Galerkin and quadrature errors committed in observables of the finite element approximation of a class of elliptic PDE with rough stochastic conductivities. These estimators, based on local error indicators, were obtained by first making an assumption on the scales of the model problem and then utilizing a simple telescoping argument. For the model problems under consideration, we demonstrated that the assumptions are satisfied. Even in the case of a simple model problem, the standard theory for constructing a posteriori error estimates failed to give reliable estimators in this setting. Therefore, the error estimates given here fill a much needed gap by providing an important and novel computational tool for this class of problems.

In this direction, these estimates are useful for constructing adaptive algorithms. These estimates are a step towards the construction of adaptive algorithms for variance reduction techniques for $\mathrm{MC}$ methods for $\mathrm{PDE}$ with rough stochastic coefficients. As mentioned in the introduction, the final level stopping criterion in a MLMC, MIMC, or CMLMC method can be built upon the error estimates derived here and a minor extension of the present theory would render it possible to also estimate mean square errors between numerical solutions on consecutive resolution levels.

\section{REFERENCES}

[1] M. Ainsworth And J. T. Oden, A posteriori error estimation in finite element analysis, Comput. Methods Appl. Mech. Engrg., 142 (1997), pp. 1-88.

[2] S. Asmussen and P. W. Glynn, Stochastic Simulation: Algorithms and Analysis, vol. 57 of Stochastic Modelling and Applied Probability, Springer, New York, 2007.

[3] I. BABUŠKA, The rate of convergence for the finite element method, SIAM J. Numer. Anal., 8 (1971), pp. 304-315.

[4] I. BABušKa AND P. Chatzipantelidis, On solving elliptic stochastic partial differential equations, Comput. Methods Appl. Mech. Engrg., 191 (2002), pp. 4093-4122.

[5] I. Babuška, F. Nobile, And R. Tempone, A stochastic collocation method for elliptic partial differential equations with random input data, SIAM J. Numer. Anal., 45 (2007), pp. 10051034 .

[6] I. BABUŠKa AND W. C. Rheinboldt, Error estimates for adaptive finite element computations, SIAM J. Numer. Anal., 15 (1978), pp. 736-754.

[7] I. BabušKa AND W. C. Rheinboldt, A posteriori error analysis of finite element solutions for one-dimensional problems, SIAM J. Numer. Anal., 18 (1981), pp. 565-589.

[8] I. BabušKa, R. Tempone, And G. E. Zouraris, Galerkin finite element approximations of stochastic elliptic partial differential equations, SIAM J. Numer. Anal., 42 (2004), pp. 800825 .

[9] W. Bangerth And R. RANnACher, Adaptive finite element methods for differential equations, Lectures in Mathematics ETH Zürich, Birkhäuser Verlag, Basel, 2003.

[10] A. Barth, C. Schwab, And N. Zollinger, Multi-level Monte Carlo finite element method for elliptic PDEs with stochastic coefficients, Numer. Math., 119 (2011), pp. 123-161.

[11] S. C. Brenner and L. R. Scott, The mathematical theory of finite element methods, vol. 15 of Texts in Applied Mathematics, Springer, New York, third ed., 2008.

[12] J. ChARRIER, Strong and weak error estimates for elliptic partial differential equations with random coefficients, SIAM J. Numer. Anal., 50 (2012), pp. 216-246.

[13] J. Charrier, R. Scheichl, And A. L. Teckentrup, Finite element error analysis of elliptic PDEs with random coefficients and its application to multilevel Monte Carlo methods, SIAM J. Numer. Anal., 51 (2013), pp. 322-352.

[14] K. A. Cliffe, M. B. Giles, R. Scheichl, And A. L. Teckentrup, Multilevel Monte Carlo methods and applications to elliptic PDEs with random coefficients, Comput. Vis. Sci., 14 (2011), pp. 3-15. 
[15] N. Collier, A.-L. Haji-Ali, F. Nobile, E. von Schwerin, and R. Tempone, A continuation multilevel monte carlo algorithm, BIT Numerical Mathematics, 55 (2014), pp. 399-432.

[16] G. DaGan, Statistical theory of groundwater flow and transport: Pore to laboratory, laboratory to formation, and formation to regional scale, Water Resources Research, 22 (1986), pp. 120S-134S.

[17] - Stochastic modeling of flow and transport: The broad perspective, in Subsurface Flow and Transport: A Stochastic Approach, G. Dagan and S. P. Neuman, eds., Cambridge University Press, 2005.

[18] G. Dagan and S. P. Neuman, eds., Subsurface Flow and Transport: A Stochastic Approach, Cambridge University Press, Cambridge, 2005.

[19] M. K. Deb, I. M. BAbuŠKa, And J. T. Oden, Solution of stochastic partial differential equations using Galerkin finite element techniques, Comput. Methods Appl. Mech. Engrg., 190 (2001), pp. 6359-6372.

[20] J. P. Delhomme, Spatial variability and uncertainty in groundwater flow parameters: A geostatistical approach, Water Resources Research, 15 (1979), pp. 269-280.

[21] M. Eigel, C. Gittelson, C. Schwab, And E. Zander, Adaptive stochastic Galerkin FEM, Tech. Report 2013-01, Seminar for Applied Mathematics, ETH Zürich, Switzerland, 2013.

[22] M. Eigel AND C. Merdon, Local equilibration error estimators for guaranteed error control in adaptive stochastic higher-order Galerkin FEM, tech. report, WIAS Preprint No. 1997, 2014.

[23] K. Eriksson, D. Estep, P. Hansbo, And C. Johnson, Introduction to adaptive methods for differential equations, in Acta numerica, 1995, Acta Numer., Cambridge Univ. Press, Cambridge, 1995, pp. 105-158.

[24] X. FERnique, Regularité des trajectoires des fonctions aléatoires gaussiennes, in École d'Été de Probabilités de Saint-Flour, IV-1974, Springer, Berlin, 1975, pp. 1-96. Lecture Notes in Math., Vol. 480.

[25] P. Frauenfelder, C. Schwab, And R. A. Todor, Finite elements for elliptic problems with stochastic coefficients, Comput. Methods Appl. Mech. Engrg., 194 (2005), pp. 205-228.

[26] M. B. GILEs, Multilevel Monte Carlo path simulation, Oper. Res., 56 (2008), pp. 607-617.

[27] M. B. Giles ANd E. SüLI, Adjoint methods for PDEs: a posteriori error analysis and postprocessing by duality, Acta Numer., 11 (2002), pp. 145-236.

[28] C. J. GitTelson, Stochastic Galerkin discretization of the log-normal isotropic diffusion problem, Math. Models Methods Appl. Sci., 20 (2010), pp. 237-263.

[29] C. J. Gittelson, An adaptive stochastic Galerkin method for random elliptic operators, Math. Comp., 82 (2013), pp. 1515-1541.

[30] I. G. Graham, R. Scheichl, and E. Ullmann, Mixed finite element analysis of lognormal diffusion and multilevel monte carlo methods. arXiv preprint, 122013.

[31] A.-L. Haji-Ali, F. Nobile, and R. Tempone, Multi-Index Monte Carlo: When sparsity meets sampling. arXiv preprint, 052014.

[32] R. J. Hoeksema and P. K. Kitanidis, Analysis of the spatial structure of properties of selected aquifers, Water Resources Research, 21 (1985), pp. 563-572.

[33] H. Hoel, J. HÄppölä, And R. Tempone, Construction of a mean square error adaptive eulermaruyama method with applications in multilevel monte carlo, submitted, (2015).

[34] I. Janković, A. Fiori, R. Suribhatla, and G. Dagan, Identification of heterogeneous aquifer transmissivity using an AE-based method, Groundwater, 44 (2006), pp. 62-71.

[35] I. Karatzas and S. E. Shreve, Brownian motion and stochastic calculus, vol. 113 of Graduate Texts in Mathematics, Springer-Verlag, New York, second ed., 1991.

[36] H. G. Matthies And A. Keese, Galerkin methods for linear and nonlinear elliptic stochastic partial differential equations, Comput. Methods Appl. Mech. Engrg., 194 (2005), pp. 12951331.

[37] K.-S. Moon, E. von Schwerin, A. Szepessy, and R. Tempone, Convergence rates for an adaptive dual weighted residual finite element algorithm, BIT, 46 (2006), pp. 367-407.

[38] A. Mugler ANd H.-J. StARKLOFF, On elliptic partial differential equations with random coefficients, Stud. Univ. Babeş-Bolyai Math., 56 (2011), pp. 473-487.

[39] A. Szepessy, R. Tempone, And G. E. Zouraris, Adaptive weak approximation of stochastic differential equations, Comm. Pure Appl. Math., 54 (2001), pp. 1169-1214.

[40] A. L. Teckentrup, R. Scheichl, M. B. Giles, and E. Ullmann, Further analysis of multilevel Monte Carlo methods for elliptic PDEs with random coefficients, Numer. Math., 125 (2013), pp. 569-600. 